\title{
Qualitative properties of singular solutions to nonlocal problems
}

\author{
Luigi Montoro ${ }^{1} \cdot$ Fabio Punzo $^{2} \cdot$ Berardino Sciunzi $^{1}$
}

Received: 19 April 2017 / Accepted: 16 October 2017 / Published online: 27 October 2017

(c) Fondazione Annali di Matematica Pura ed Applicata and Springer-Verlag GmbH Germany 2017

\begin{abstract}
We consider positive weak solutions to $(-\Delta)^{s} u=f(x, u)$ in $\Omega \backslash \Gamma$ under zero Dirichlet boundary condition. The domain $\Omega$ is bounded or is the whole space, and the solution has a singularity on the singular set $\Gamma$. Under suitable assumptions on $f$ we prove symmetry and monotonicity properties of the solutions when the singular set $\Gamma$ has zero $s$-capacity.
\end{abstract}

Keywords Fractional Laplacian · Qualitative properties · Moving plane method

Mathematics Subject Classification $35 \mathrm{R} 09 \cdot 34 \mathrm{~B} 10 \cdot 35 \mathrm{~B} 06 \cdot 35 \mathrm{~B} 51$

\section{Introduction}

In this paper we study the following nonlocal semilinear elliptic problem:

$$
\left\{\begin{aligned}
(-\Delta)^{s} u & =f(x, u) & & \text { in } \Omega \backslash \Gamma, \\
u & >0 & & \text { in } \Omega \backslash \Gamma, \\
u & =0 & & \text { in } \mathbb{R}^{N} \backslash \Omega,
\end{aligned}\right.
$$

The authors are partially supported by the Gnampa Project 2016 'Proprietà qualitative di equazioni ellittiche e paraboliche non lineari'.

Berardino Sciunzi

sciunzi@mat.unical.it

Luigi Montoro

montoro@mat.unical.it

Fabio Punzo

fabio.punzo@polimi.it

1 Dipartimento di Matematica e Informatica, Università della Calabria, Ponte Pietro Bucci 31B, Arcavacata di Rende, 87036 Cosenza, Italy

2 Dipartimento di Matematica, Politecnico di Milano, via E. Bonardi 9, 20133 Milan, Italy 
where $0<s<1, N>2 s$ and $\Omega$ is a bounded domain with smooth boundary $\partial \Omega$, or it is the whole space $\mathbb{R}^{N}$. Note that the equation is satisfied in $\Omega \backslash \Gamma$, where the set $\Gamma \subset \Omega$, which is referred to as the singular set, is compact and has zero $s$-capacity (see Sect. 2 below). We consider solutions belonging to $W_{\text {loc }}^{s, 2}\left(\mathbb{R}^{N} \backslash \Gamma\right) \cap L^{1}\left(\mathbb{R}^{N}\right) \cap C\left(\mathbb{R}^{N} \backslash \Gamma\right)$, and the equation is understood in the weak distributional sense, see Definition 2.2 below. As it is customary, in the case of a bounded domain $\Omega$, the Dirichlet datum is expressed by the fact that $u$ is identically zero outside $\Omega$.

We study symmetry and monotonicity properties of solutions via the moving plane method that was introduced in $[2,22]$, and in particular we refer to the celebrated papers $[4,15]$ where it was firstly exploited to study symmetry and monotonicity properties of the solutions.

Here we deal with singular solutions in the nonlocal case; for the local case we refer to $[6,21,23,28]$. Symmetry results, when $\Gamma=\emptyset$, for equations involving the fractional Laplacian via the moving plane method, for more regular problems, can be found for instance in $[3,12,16,17]$ and also in $[7,8,12,14,20]$. Other works, for the case $\Gamma=\emptyset$ and in the nonlocal framework, that study the symmetry of solutions using other techniques are, for example, [5,11,24].

In our results we shall assume in the case of a bounded domain $\Omega$ that the nonlinearity $f$ is uniformly locally Lipschitz continuous far from the singular set $\Gamma$. More precisely we make the following assumption:

$\left(A_{f}^{1}\right)$. For any $0 \leq \tau, t \leq M$ and for any compact set $K \subset \Omega \backslash \Gamma$, there exists a positive constant $C=C(K, M)$ such that

$$
|f(x, \tau)-f(x, t)| \leq C|\tau-t| \text { for any } x \in K .
$$

Furthermore, $f(\cdot, \tau)$ is nondecreasing in the $x_{1}$-direction in $\Omega \cap\left\{x_{1}<0\right\}$ and symmetric with respect to the hyperplane $\left\{x_{1}=0\right\}$.

In this setting our main result is the following

Theorem 1.1 Let $u \in W_{\text {loc }}^{s, 2}\left(\mathbb{R}^{N} \backslash \Gamma\right) \cap L^{1}\left(\mathbb{R}^{N}\right) \cap C\left(\mathbb{R}^{N} \backslash \Gamma\right)$ be a solution to (1.1) with $f$ fulfilling $\left(A_{f}^{1}\right)$. Assume that the singular set $\Gamma \subset \Omega$ is compact and has zero s-capacity.

If $\Omega$ is convex and symmetric in the $x_{1}$-direction and $\Gamma \subset\left\{x_{1}=0\right\}$, then $u$ is symmetric with respect to the hyperplane $\left\{x_{1}=0\right\}$ and increasing in the $x_{1}$-direction in $\Omega \cap\left\{x_{1}<0\right\}$.

If the domain is a ball and $\Gamma$ is the center of the ball, then the solution is radial and radially decreasing about the center of the ball.

The proof exploits a new technique based also on some ideas introduced in [23] for the local case. The nonlocal case exhibits many peculiarities related in particular to the notion of solution and to the fact that the critical set plays a role also far from it, because of the nonlocal nature of the operator.

In the second part of the paper we consider problem (1.1), with $f=f(u)$ in the whole space $\mathbb{R}^{N}$, that is we consider

$$
\left\{\begin{array}{l}
(-\Delta)^{s} u=f(u) \quad \text { in } \mathbb{R}^{N} \backslash \Gamma, \\
u>0 \quad \text { in } \mathbb{R}^{N} \backslash \Gamma,
\end{array}\right.
$$

with $f(\cdot)$ satisfying a critical growth assumption, namely:

$\left(A_{f}^{2}\right) f$ is $C^{1}$ and convex with $f(0)=0$ and, for any $t>0$

$$
f^{\prime}(t) \leq C_{f} t^{2_{s}^{*}-2},
$$

for some $C_{f}>0$, where $2_{s}^{*}=2 N /(N-2 s), N>2 s$ is the Sobolev critical exponent. We dropped the dependence of $f$ on $x$ to avoid further technicalities. 
In this setting our main result is the following

Theorem 1.2 Let $u \in W_{\text {loc }}^{s, 2}\left(\mathbb{R}^{N} \backslash \Gamma\right) \cap L^{1}\left(\mathbb{R}^{N}\right) \cap C\left(\mathbb{R}^{N} \backslash \Gamma\right)$ be a solution to (1.2) with $f$ fulfilling $\left(A_{f}^{2}\right)$. Assume that the singular set $\Gamma \subset \mathbb{R}^{N}$ is compact and has zero s-capacity.

If for some $R_{0}>0, \Gamma \subset\left\{x_{1}=0\right\} \cap B_{R_{0}}$ and $u \in L^{2_{s}^{*}}\left(\mathbb{R}^{N} \backslash B_{R_{0}}\right)$, then $u$ is symmetric with respect to the hyperplane $\left\{x_{1}=0\right\}$ and increasing in the $x_{1}$-direction in $\left\{x_{1}<0\right\}$.

If $u$ has only a nonremovable singularity at the origin, then the solution is radial and radially decreasing about the origin.

In the local case the problem in the whole space can be studied in a similar way as in the case of a bounded domain. This is not the case when considering nonlocal problems; indeed, a fine density argument and new estimates are required.

The paper is organized as follows: we collect some preliminary results in Sect. 2. The case of a bounded domain, namely Theorem 1.1, is studied in Sect. 3. In Sect. 4 we deal with the case of the whole space and we prove Theorem 1.2.

\section{Notations and preliminary results}

Let us recall that, given a function $u$ in the Schwartz's class $\mathcal{S}\left(\mathbb{R}^{N}\right)$ we define for $0<s<1$, the fractional Laplacian as

$$
\widehat{(-\Delta)^{s}} u(\xi)=|\xi|^{2 s} \widehat{u}(\xi), \quad \xi \in \mathbb{R}^{N},
$$

where $\widehat{u} \equiv \mathfrak{F}(u)$ is the Fourier transform of $u$. It is well known (see $[18,27,29]$ ) that this operator can be also represented, for suitable functions, as a principal value of the form

$$
(-\Delta)^{s} u(x):=c_{N, s} \text { P.V. } \int_{\mathbb{R}^{N}} \frac{u(x)-u(y)}{|x-y|^{N+2 s}} \mathrm{~d} y
$$

where

$$
c_{N, s}:=\left(\int_{\mathbb{R}^{N}} \frac{1-\cos \left(\xi_{1}\right)}{|\xi|^{N+2 s}} d \xi\right)^{-1}=\frac{4^{s} \Gamma\left(\frac{N}{2}+s\right)}{-\pi^{\frac{N}{2}} \Gamma(-s)}>0,
$$

is a normalizing constant chosen to guarantee that (2.1) is satisfied (see $[9,25,29])$. From (2.2) one can check that

$$
\left|(-\Delta)^{s} \phi(x)\right| \leq \frac{C}{1+|x|^{N+2 s}}, \quad \text { for every } \phi \in \mathcal{S}\left(\mathbb{R}^{N}\right) .
$$

This motivates the introduction of the space

$$
\mathcal{L}^{s}\left(\mathbb{R}^{N}\right):=\left\{u: \mathbb{R}^{N} \rightarrow \mathbb{R}: \quad \int_{\mathbb{R}^{N}} \frac{|u(x)|}{1+|x|^{N+2 s}} \mathrm{~d} x<\infty\right\},
$$

endowed with the natural norm

$$
\|u\|_{\mathcal{L}^{s}\left(\mathbb{R}^{N}\right)}:=\int_{\mathbb{R}^{N}} \frac{|u(x)|}{1+|x|^{N+2 s}} \mathrm{~d} x .
$$

Then, if $u \in \mathcal{L}^{s}\left(\mathbb{R}^{N}\right)$ and $\phi \in \mathcal{S}\left(\mathbb{R}^{N}\right)$, using (2.4), we can formally define the duality product $\left\langle(-\Delta)^{s} u, \phi\right\rangle$ in the distributional sense as

$$
\left\langle(-\Delta)^{s} u, \phi\right\rangle:=\int_{\mathbb{R}^{N}} u(-\Delta)^{s} \phi \mathrm{d} x .
$$


We consider the Sobolev space

$$
H^{s}\left(\mathbb{R}^{N}\right):=\left\{u \in L^{2}\left(\mathbb{R}^{N}\right):|\zeta|^{s} \hat{u} \in L^{2}\left(\mathbb{R}^{N}\right)\right\}
$$

endowed with the norm

$$
\|u\|_{H^{s}\left(\mathbb{R}^{N}\right)}:=\|\hat{u}\|_{L^{2}\left(\mathbb{R}^{N}\right)}+\left\|\zeta^{S} \hat{u}\right\|_{L^{2}\left(\mathbb{R}^{N}\right)} .
$$

We also consider the Hilbert space $\mathfrak{D}^{s, 2}\left(\mathbb{R}^{N}\right)$, which is the completion of $C_{c}^{\infty}\left(\mathbb{R}^{N}\right)$ w.r.t. the norm

$$
\left\||\zeta|^{s} \hat{u}\right\|_{L^{2}\left(\mathbb{R}^{N}\right)}=\frac{2}{c_{N, s}}\left\|(-\Delta)^{s / 2} u\right\|_{L^{2}\left(\mathbb{R}^{N}\right)}^{2} .
$$

Furthermore, for any open subset $\Omega \subseteq \mathbb{R}^{N}$ with smooth boundary $\partial \Omega$, and for any $p>1$ let $W^{s, p}(\Omega)$ be the space of measurable functions $u: \Omega \rightarrow \mathbb{R}$ such that the norm

$$
\|u\|_{W^{s, p}(\Omega)}^{p}:=\|u\|_{L^{p}(\Omega)}^{p}+\int_{\Omega} \int_{\Omega} \frac{|u(x)-u(y)|^{p}}{|x-y|^{N+p s}} \mathrm{~d} x \mathrm{~d} y
$$

is finite. In addition, denote by $W_{0}^{s, p}(\Omega)$ the closure of $C_{c}^{\infty}(\Omega)$ with respect to the norm $\|\cdot\|_{W^{s, p}(\Omega)}$. We set

$$
H^{s}(\Omega) \equiv W^{s, 2}(\Omega), H_{0}^{s}(\Omega) \equiv W_{0}^{s, 2}(\Omega) .
$$

Moreover, we say that $u \in W_{\text {loc }}^{s, 2}(\Omega)$, if for every compact subset $K \subset \Omega$ we have that $u \in W^{s, 2}(K)$. We also set

$$
\mathcal{H}_{0}^{s}(\Omega):=\left\{u \in H^{s}(\Omega): \tilde{u} \in \mathfrak{D}^{s, 2}\left(\mathbb{R}^{N}\right)\right\},
$$

where

$$
\tilde{u}:= \begin{cases}u & \text { in } \Omega, \\ 0 & \text { in } \mathbb{R}^{N} \backslash \Omega .\end{cases}
$$

$\mathcal{H}_{0}^{s}(\Omega)$, equipped with the norm

$$
\|u\|_{\mathcal{H}_{0}^{s}(\Omega)}^{2}:=\int_{\mathbb{R}^{N}}|\zeta|^{2 s}|\mathfrak{F}(\tilde{u})|^{2} \mathrm{~d} \zeta,
$$

is a Hilbert space. If $\Omega$ is bounded (see, for example, [13]), then there exists a constant $C=C(\Omega)>0$ such that

$$
C\|\tilde{u}\|_{H^{s}\left(\mathbb{R}^{N}\right)} \leq\|u\|_{\mathcal{H}_{0}^{s}(\Omega)} \leq\|\tilde{u}\|_{H^{s}\left(\mathbb{R}^{N}\right)} \text { for any } u \in \mathcal{H}_{0}^{s}(\Omega) .
$$

Thus,

$$
\mathcal{H}_{0}^{s}(\Omega)=\left\{u \in H^{s}(\Omega): \tilde{u} \in H^{s}\left(\mathbb{R}^{N}\right)\right\} .
$$

Moreover, $C_{c}^{\infty}(\Omega)$ is dense in $\mathcal{H}_{0}^{s}(\Omega)$.

In the following we will exploit the following well known Sobolev-type embedding Theorem

Theorem 2.1 (See [1, Theorem 7.58], [9, Theorem 6.5], [19,26]) Let $0<s<1$ and $N>2 s$. There exists a constant $S_{N, s}$ such that, for any measurable and compactly supported function $u: \mathbb{R}^{N} \rightarrow \mathbb{R}$, we have

$$
S_{N, s}\|u\|_{L^{2 *}\left(\mathbb{R}^{N}\right)}^{2} \leq \frac{2}{c_{N, s}}\left\|(-\Delta)^{s / 2} u\right\|_{L^{2}\left(\mathbb{R}^{N}\right)}^{2},
$$


where

$$
2_{s}^{*}=\frac{2 N}{N-2 s}
$$

is the Sobolev critical exponent.

Now we are in position to give the following

Definition 2.2 We say that $u \in W_{\text {loc }}^{s, 2}\left(\mathbb{R}^{N} \backslash \Gamma\right) \cap L^{1}\left(\mathbb{R}^{N}\right)$ is a weak solution to (1.1) if

$$
u=0 \text { in } \mathbb{R}^{N} \backslash \Omega
$$

and

$$
\begin{aligned}
& \frac{1}{2} c_{N, s} \int_{\mathbb{R}^{N}} \int_{\mathbb{R}^{N}} \frac{(u(x)-u(y))(\varphi(x)-\varphi(y))}{|x-y|^{N+2 s}} \mathrm{~d} x \mathrm{~d} y \\
& =\int_{\mathbb{R}^{N}} f(x, u) \varphi \mathrm{d} x \quad \forall \varphi \in C_{c}^{\infty}(\Omega \backslash \Gamma) .
\end{aligned}
$$

where $c_{N, s}$ has been defined in (2.3).

For the reader's convenience, in order to show that Definition 2.2 is well posed, we prove the following

Proposition 2.3 Let $u \in W_{\mathrm{loc}}^{s, 2}\left(\mathbb{R}^{N} \backslash \Gamma\right) \cap L^{1}\left(\mathbb{R}^{N}\right)$. Then, for any $\varphi \in C_{c}^{\infty}(\Omega \backslash \Gamma)$,

$$
\frac{1}{2} c_{N, s} \int_{\mathbb{R}^{N}} \int_{\mathbb{R}^{N}} \frac{(u(x)-u(y))(\varphi(x)-\varphi(y))}{|x-y|^{N+2 s}} \mathrm{~d} x \mathrm{~d} y<\infty .
$$

Proof Let $\varphi \in C_{c}^{\infty}(\Omega \backslash \Gamma)$ and let us denote $K_{\varphi}=\operatorname{supp}(\varphi)$. Fix now a compact set $K \subset \Omega \backslash \Gamma$ such that $K_{\varphi} \subset K$ and use the decomposition

$$
\mathbb{R}^{N} \times \mathbb{R}^{N}=\left(K \cup K^{c}\right) \times\left(K \cup K^{c}\right),
$$

where $K^{c}:=\mathbb{R}^{N} \backslash K$. Thus,

$$
\begin{aligned}
& \frac{1}{2} c_{N, s} \int_{\mathbb{R}^{N}} \int_{\mathbb{R}^{N}} \frac{(u(x)-u(y))(\varphi(x)-\varphi(y))}{|x-y|^{N+2 s}} \mathrm{~d} x \mathrm{~d} y \\
& =\frac{1}{2} c_{N, s} \int_{K} \int_{K} \frac{(u(x)-u(y))(\varphi(x)-\varphi(y))}{|x-y|^{N+2 s}} \mathrm{~d} x \mathrm{~d} y \\
& \quad+\frac{1}{2} c_{N, s} \int_{K} \int_{K^{c}} \frac{(u(x)-u(y))(\varphi(x)-\varphi(y))}{|x-y|^{N+2 s}} \mathrm{~d} x \mathrm{~d} y \\
& \quad+\frac{1}{2} c_{N, s} \int_{K^{c}} \int_{K} \frac{(u(x)-u(y))(\varphi(x)-\varphi(y))}{|x-y|^{N+2 s}} \mathrm{~d} x \mathrm{~d} y,
\end{aligned}
$$

since

$$
\frac{1}{2} c_{N, s} \int_{K^{c}} \int_{K^{c}} \frac{(u(x)-u(y))(\varphi(x)-\varphi(y))}{|x-y|^{N+2 s}} \mathrm{~d} x \mathrm{~d} y=0 .
$$

We prove that all the three terms on the right-hand side of (2.7) are finites. In fact

$$
\frac{1}{2} c_{N, s} \int_{K} \int_{K} \frac{(u(x)-u(y))(\varphi(x)-\varphi(y))}{|x-y|^{N+2 s}} \mathrm{~d} x \mathrm{~d} y<C,
$$

for some positive constant $C$, since by hypothesis $u \in W_{\text {loc }}^{s, 2}\left(\mathbb{R}^{N} \backslash \Gamma\right)$ and $K \subset \Omega \backslash \Gamma$. Therefore, by Hölder inequality, (2.8) follows. 
We can write the second term as

$$
\begin{aligned}
& \frac{1}{2} c_{N, s} \int_{K} \int_{K^{c}} \frac{(u(x)-u(y))(\varphi(x)-\varphi(y))}{|x-y|^{N+2 s}} \mathrm{~d} x \mathrm{~d} y \\
& \quad=\frac{1}{2} c_{N, s} \int_{K_{\varphi}} \int_{K^{c}} \frac{(u(x)-u(y))(\varphi(x)-\varphi(y))}{|x-y|^{N+2 s}} \mathrm{~d} x \mathrm{~d} y .
\end{aligned}
$$

We observe that, for all points $(x, y) \in K_{\varphi} \times K^{c}$, we have that $|x-y| \geq \delta>0$, for some positive constant $\delta=\delta\left(K, K_{\varphi}\right)$. We deduce

$$
\frac{1}{2} c_{N, s} \int_{K_{\varphi}} \int_{K^{c}} \frac{(u(x)-u(y))(\varphi(x)-\varphi(y))}{|x-y|^{N+2 s}} \mathrm{~d} x \mathrm{~d} y \leq C,
$$

with $C=C\left(\delta, K, K_{\varphi},\|u\|_{L^{1}\left(\mathbb{R}^{N}\right)},\|\varphi\|_{L^{\infty}\left(K_{\varphi}\right)}\right)$ a positive constant. Here we have used the fact that $u \in L^{1}\left(\mathbb{R}^{N}\right)$ and $\varphi \in C^{\infty}\left(K_{\varphi}\right)$. From (2.9) and (2.10) we obtain

$$
\frac{1}{2} c_{N, s} \int_{K} \int_{K^{c}} \frac{(u(x)-u(y))(\varphi(x)-\varphi(y))}{|x-y|^{N+2 s}} \mathrm{~d} x \mathrm{~d} y \leq C .
$$

For the third term we argue in the same way as in (2.9), (2.10) and (2.11). Finally, by (2.7) we obtain the thesis.

For future use we point out the following

Lemma 2.4 Let $u \in W_{\text {loc }}^{s, 2}\left(\mathbb{R}^{N} \backslash \Gamma\right) \cap L^{1}\left(\mathbb{R}^{N}\right)$ be a weak solution to (1.1), according to Definition 2.2. Then,

$$
\frac{1}{2} c_{N, s} \int_{\mathbb{R}^{N}} \int_{\mathbb{R}^{N}} \frac{(u(x)-u(y))(\varphi(x)-\varphi(y))}{|x-y|^{N+2 s}} \mathrm{~d} x \mathrm{~d} y=\int_{\mathbb{R}^{N}} f(x, u) \varphi \mathrm{d} x
$$

for any $\varphi \in W_{0}^{s, 2}(\Omega \backslash \Gamma)$ with compact support in $\Omega \backslash \Gamma$.

Proof For any $\varphi \in W_{0}^{s, 2}(\Omega \backslash \Gamma)$ with compact support in $\Omega \backslash \Gamma$, by a convolution argument, we can consider a sequence of functions $\varphi_{n}$ with compact support still in $\Omega \backslash \Gamma$ such that

$$
\varphi_{n} \in C_{c}^{\infty}(\Omega \backslash \Gamma) \text { and } \varphi_{n} \stackrel{W_{0}^{s, 2}(\Omega)}{\rightarrow} \varphi .
$$

Plugging $\varphi_{n}$ as test function in (1.1) and passing to the limit we obtain the thesis. It is crucial here the fact that, by the properties of the convolution, we can assume that the supports of the functions involved remain bounded away from the singular set.

For any given compact subset $\Gamma \subset \Omega$ we define the relative s-capacity of $\Gamma$ w.r.t. $\Omega$ as follows (see, for example, [13]):

$$
\operatorname{Cap}_{s}^{\Omega}(\Gamma):=\inf _{\phi \in C_{c}^{\infty}(\Omega)}\left\{\|\phi\|_{\mathcal{H}_{0}^{s}(\Omega)}^{2}: \phi \geq 1 \text { in a neighborhood of } \Gamma\right\} .
$$

Moreover, we define the $s$-capacity of $\Gamma$ by

$$
\operatorname{Cap}_{s}(\Gamma):=\inf _{\phi \in C_{c}^{\infty}\left(\mathbb{R}^{N}\right)}\left\{\|\phi\|_{\mathfrak{D}^{s, 2}\left(\mathbb{R}^{N}\right)}^{2}: \phi \geq 1 \text { in a neighborhood of } \Gamma\right\} .
$$

We have the next result.

Lemma 2.5 Let $\Omega \subset \mathbb{R}^{N}$ be an open bounded subset; let $\Gamma \subset \Omega$ be a compact subset. Then, there exists a constant $K>1$ such that

$$
\operatorname{Cap}_{s}(\Gamma) \leq \operatorname{Cap}_{s}^{\Omega}(\Gamma) \leq K \operatorname{Cap}_{s}(\Gamma) \text {. }
$$


Note that an estimate similar to (2.14) is established in [30]; however, in [30] a slightly different definition of $s$-capacity is used. Moreover, the relation between the $s$-capacity and the Haussdorf measure is described also with various examples.

Proof In view of (2.12) and (2.13), clearly, we have that

$$
\operatorname{Cap}_{s}(\Gamma) \leq \operatorname{Cap}_{s}^{\Omega}(\Gamma) .
$$

Note that, due to (2.13), for any $\epsilon>0$ there exists $\phi_{\epsilon} \in C_{c}^{\infty}\left(\mathbb{R}^{N}\right)$ such that

$$
\left\|\phi_{\epsilon}\right\|_{\mathfrak{D}^{s, 2}\left(\mathbb{R}^{N}\right)}^{2} \leq \operatorname{Cap}_{s}(\Gamma)+\epsilon .
$$

We can select (see [9]) an open subset $\Omega^{\prime} \subset \subset \Omega$ and a function $\eta_{\epsilon} \in W^{2, s}\left(\mathbb{R}^{N}\right)$ such that

$$
\begin{aligned}
& \eta_{\epsilon}=\phi_{\epsilon} \text { in } \Omega^{\prime}, \\
& \eta_{\epsilon}=0 \text { in } \mathbb{R}^{N} \backslash \Omega .
\end{aligned}
$$

Moreover, we can find a constant $\tilde{C}=\tilde{C}\left(\Omega^{\prime}\right)>0$ such that

$$
\left\|\eta_{\epsilon}\right\|_{W^{2, s}\left(\mathbb{R}^{N}\right)} \leq \tilde{C}\left\|\eta_{\epsilon}\right\|_{W^{2, s}\left(\Omega^{\prime}\right)} .
$$

Note that thanks to (2.18), we have that $\eta_{\epsilon} \in \mathcal{H}_{0}^{s}(\Omega)$. Using the fact that $C_{c}^{\infty}(\Omega)$ is dense in $\mathcal{H}_{0}^{s}(\Omega),(2.19)$, and Theorem 2.1, we can infer that

$$
\begin{aligned}
\operatorname{Cap}_{s}^{\Omega}(\Gamma) & \leq\left\|\eta_{\epsilon}\right\|_{\mathfrak{D}^{s, 2}\left(\mathbb{R}^{N}\right)}^{2} \leq\left\|\eta_{\epsilon}\right\|_{W^{s, 2}\left(\mathbb{R}^{N}\right)}^{2} \leq \tilde{C}^{2}\left\|\eta_{\epsilon}\right\|_{W^{s, 2}\left(\Omega^{\prime}\right)}^{2} \\
& \leq C\left[\left\|\phi_{\epsilon}\right\|_{L^{2}(\Omega)}^{2}+\left\|\phi_{\epsilon}\right\|_{\mathfrak{D}^{s, 2}\left(\mathbb{R}^{N}\right)}^{2}\right] \\
& \leq C\left[\left\|\phi_{\epsilon}\right\|_{L^{2 *}(\Omega)}^{2}+\left\|\phi_{\epsilon}\right\|_{\mathfrak{D}^{s, 2}\left(\mathbb{R}^{N}\right)}^{2}\right] \leq C\left(\operatorname{Cap}_{s}(\Gamma)+\epsilon\right),
\end{aligned}
$$

for some positive constant $C$ independent of $\epsilon$. Letting $\epsilon \rightarrow 0^{+}$, we get

$$
\operatorname{Cap}_{s}^{\Omega}(\Gamma) \leq \operatorname{Cap}_{s}(\Gamma) .
$$

This combined with (2.12) yields (2.14). The proof is complete.

We will use the following notations. For a real number $\lambda \leq 0$ we set

$$
\begin{aligned}
\Omega_{\lambda} & =\left\{x \in \Omega: x_{1}<\lambda\right\} \\
\Sigma_{\lambda} & =\left\{x \in \mathbb{R}^{N}: x_{1}<\lambda\right\} \\
R_{\lambda}(x) & =x_{\lambda}=\left(2 \lambda-x_{1}, x_{2}, \ldots, x_{n}\right)
\end{aligned}
$$

which is the reflection trough the hyperplane $T_{\lambda}$ and

$$
u_{\lambda}(x)=u\left(x_{\lambda}\right) .
$$

Also we define

$$
a=\inf _{x \in \Omega} x_{1}
$$

Notation. Generic fixed and numerical constants will be denoted by $C$ (with subscript in some case), and they will be allowed to vary within a single line or formula. By $|A|$ we will denote the Lebesgue measure of a measurable set $A$. 


\section{Proof of Theorem 1.1}

For $\lambda<0$ we introduce the following function

$$
w_{\lambda}(x):= \begin{cases}\left(u-u_{\lambda}\right)^{+}(x), & \text { if } x \in \Sigma_{\lambda} \\ \left(u-u_{\lambda}\right)^{-}(x), & \text { if } x \in \mathbb{R}^{N} \backslash \Sigma_{\lambda}\end{cases}
$$

where $\left(u-u_{\lambda}\right)^{+}:=\max \left\{u-u_{\lambda}, 0\right\}$ and $\left(u-u_{\lambda}\right)^{-}:=\min \left\{u-u_{\lambda}, 0\right\}$. We set

$$
\begin{array}{ll}
\mathcal{S}_{\lambda}:=\operatorname{supp} w_{\lambda}(x) \cap \Sigma_{\lambda}, & \mathcal{S}_{\lambda}^{c}:=\Sigma_{\lambda} \backslash \mathcal{S}_{\lambda}, \\
\mathcal{D}_{\lambda}:=\operatorname{supp} w_{\lambda}(x) \cap\left(\mathbb{R}^{N} \backslash \Sigma_{\lambda}\right), & \mathcal{D}_{\lambda}^{c}:=\left(\mathbb{R}^{N} \backslash \Sigma_{\lambda}\right) \backslash \mathcal{D}_{\lambda} .
\end{array}
$$

It is not difficult to see that

$$
\mathcal{D}_{\lambda} \text { is the reflection of } \mathcal{S}_{\lambda} \text {. }
$$

Lemma 3.1 Under the assumptions of Theorem 1.1 and for $a<\lambda<0$, we have that

$$
\int_{\mathbb{R}^{N}} \int_{\mathbb{R}^{N}} \frac{\left(w_{\lambda}(x)-w_{\lambda}(y)\right)^{2}}{|x-y|^{N+2 s}} \mathrm{~d} x \mathrm{~d} y \leq C\left(f, s, N,\|u\|_{L^{\infty}\left(\Omega_{\lambda}\right)}\right) .
$$

Consequently $w_{\lambda} \in \mathcal{H}_{0}^{s}\left(\Omega_{\lambda} \cup R_{\lambda}\left(\Omega_{\lambda}\right)\right)$.

Proof We start by exploiting the fact that the singular set $\Gamma$ has zero $s$-capacity. For each $\varepsilon>0$, let

$$
\Gamma_{\varepsilon}^{\lambda}:=\left\{x \in \mathbb{R}^{N} \mid \operatorname{dist}\left(x, R_{\lambda}(\Gamma)\right)<\varepsilon\right\}
$$

In view of Lemma 2.5, we have that, for each $\varepsilon>0, \operatorname{Cap}_{s}^{\Gamma_{\varepsilon}^{\lambda}}\left(R_{\lambda}(\Gamma)\right)=0$. Hence, we can find $\phi_{\varepsilon} \in C_{c}^{\infty}\left(\Gamma_{\varepsilon}^{\lambda}\right)$ such that

$$
\int_{\mathbb{R}^{N}} \int_{\mathbb{R}^{N}} \frac{\left(\phi_{\varepsilon}(x)-\phi_{\varepsilon}(y)\right)^{2}}{|x-y|^{N+2 s}} \mathrm{~d} x \mathrm{~d} y \leq \varepsilon,
$$

with $\phi_{\varepsilon} \geq 1$ on a neighborhood of $R_{\lambda}(\Gamma)$. Via a truncation argument it follows that we can assume $0 \leq \phi_{\varepsilon} \leq 1, \phi_{\varepsilon} \in \mathcal{H}_{0}^{s}\left(\Gamma_{\varepsilon}^{\lambda}\right)$. Let now

$$
g(t):=\min \{1 ; \max \{0 ; 2 t-1\}\} \quad t \in \mathbb{R},
$$

and consider

$$
\varphi_{\varepsilon}^{\lambda}(x):= \begin{cases}g\left(1-\phi_{\varepsilon}(x)\right) & \text { in } \Gamma_{\varepsilon}^{\lambda} \\ 1 & \text { in } \Sigma_{\lambda} \backslash \Gamma_{\varepsilon}^{\lambda} .\end{cases}
$$

Moreover, we extend $\varphi_{\varepsilon}^{\lambda}$ by even reflection in $\mathbb{R}^{N} \backslash \Sigma_{\lambda}$, namely $\varphi_{\varepsilon}^{\lambda}(x)=\varphi_{\varepsilon}^{\lambda}\left(x_{\lambda}\right)$ for every $x \in \mathbb{R}^{n} \backslash \Sigma_{\lambda}$. In the following, for simplicity, we use the notation $\varphi_{\varepsilon}^{\lambda}=\varphi_{\varepsilon}$. Then, we set

$$
\varphi:=w_{\lambda} \varphi_{\varepsilon}^{2}
$$

It is easy to check that

$$
(-\Delta)^{s} u_{\lambda}=f\left(x_{\lambda}, u_{\lambda}\right) \text { in } \mathbb{R}^{N} \backslash R_{\lambda}(\Gamma),
$$


in the sense of Definition 2.2. By density arguments (see Lemma 2.4), we can plug $\varphi$ as test function in Eq. (1.1) fulfilled by $u$, and in Eq. (3.7) fulfilled by $u_{\lambda}$. Arguing in this way and subtracting, we get

$$
\begin{aligned}
& \frac{1}{2} c_{N, s} \int_{\mathbb{R}^{N}} \int_{\mathbb{R}^{N}} \frac{\left(\left(u(x)-u_{\lambda}(x)\right)-\left(u(y)-u_{\lambda}(y)\right)\right)\left(w_{\lambda}(x) \varphi_{\varepsilon}^{2}(x)-w_{\lambda}(y) \varphi_{\varepsilon}^{2}(y)\right)}{|x-y|^{N+2 s}} \mathrm{~d} x \mathrm{~d} y \\
& \quad \leq \int_{\Omega_{\lambda}}\left(\frac{f(x, u)-f\left(x, u_{\lambda}\right)}{u-u_{\lambda}}\right) w_{\lambda}^{2} \varphi_{\varepsilon}^{2} \mathrm{~d} x,
\end{aligned}
$$

where we also used the monotonicity properties of $f(\cdot, u)$.

Claim: Now we claim that

$$
\begin{aligned}
& \frac{1}{2} c_{N, s} \int_{\mathbb{R}^{N}} \int_{\mathbb{R}^{N}} \frac{\left(\left(u(x)-u_{\lambda}(x)\right)-\left(u(y)-u_{\lambda}(y)\right)\right)\left(w_{\lambda}(x) \varphi_{\varepsilon}^{2}(x)-w_{\lambda}(y) \varphi_{\varepsilon}^{2}(y)\right)}{|x-y|^{N+2 s}} \mathrm{~d} x \mathrm{~d} y \\
& \quad \geq \frac{1}{2} c_{N, s} \int_{\mathbb{R}^{N}} \int_{\mathbb{R}^{N}} \frac{\left(w_{\lambda}(x)-w_{\lambda}(y)\right)\left(w_{\lambda}(x) \varphi_{\varepsilon}^{2}(x)-w_{\lambda}(y) \varphi_{\varepsilon}^{2}(y)\right)}{|x-y|^{N+2 s}} \mathrm{~d} x \mathrm{~d} y
\end{aligned}
$$

To prove this we follow closely the technique in [12] and we argue as follows. We have that

$$
\begin{aligned}
& \int_{\mathbb{R}^{N}} \int_{\mathbb{R}^{N}} \frac{\left(\left(u(x)-u_{\lambda}(x)\right)-\left(u(y)-u_{\lambda}(y)\right)\right)\left(w_{\lambda}(x) \varphi_{\varepsilon}^{2}(x)-w_{\lambda}(y) \varphi_{\varepsilon}^{2}(y)\right)}{|x-y|^{N+2 s}} \mathrm{~d} x \mathrm{~d} y \\
& \quad=\int_{\mathbb{R}^{N}} \int_{\mathbb{R}^{N}} \frac{\left(w_{\lambda}(x)-w_{\lambda}(y)\right)\left(w_{\lambda}(x) \varphi_{\varepsilon}^{2}(x)-w_{\lambda}(y) \varphi_{\varepsilon}^{2}(y)\right)}{|x-y|^{N+2 s}} \mathrm{~d} x \mathrm{~d} y \\
& \quad+\int_{\mathbb{R}^{N}} \int_{\mathbb{R}^{N}} \frac{\left(\left(u(x)-u_{\lambda}(x)\right)-\left(u(y)-u_{\lambda}(y)\right)-\left(w_{\lambda}(x)-w_{\lambda}(y)\right)\right)\left(w_{\lambda}(x) \varphi_{\varepsilon}^{2}(x)-w_{\lambda}(y) \varphi_{\varepsilon}^{2}(y)\right)}{|x-y|^{N+2 s}} \mathrm{~d} x \mathrm{~d} y \\
& =\int_{\mathbb{R}^{N}} \int_{\mathbb{R}^{N}} \frac{\left(w_{\lambda}(x)-w_{\lambda}(y)\right)\left(w_{\lambda}(x) \varphi_{\varepsilon}^{2}(x)-w_{\lambda}(y) \varphi_{\varepsilon}^{2}(y)\right)}{|x-y|^{N+2 s}} \mathrm{~d} x \mathrm{~d} y+\int_{\mathbb{R}^{N}} \int_{\mathbb{R}^{N}} \frac{\mathcal{G}(x, y)}{|x-y|^{N+2 s}} \mathrm{~d} x \mathrm{~d} y,
\end{aligned}
$$

where

$$
\mathcal{G}(x, y):=\left(\left(u(x)-u_{\lambda}(x)\right)-\left(u(y)-u_{\lambda}(y)\right)-\left(w_{\lambda}(x)-w_{\lambda}(y)\right)\right)\left(w_{\lambda}(x) \varphi_{\varepsilon}^{2}(x)-w_{\lambda}(y) \varphi_{\varepsilon}^{2}(x)\right) .
$$

Now, we prove that

$$
\int_{\mathbb{R}^{N}} \int_{\mathbb{R}^{N}} \frac{\mathcal{G}(x, y)}{|x-y|^{N+2 s}} \mathrm{~d} x \mathrm{~d} y \geq 0 .
$$

To check this, we use the decomposition

$$
\mathbb{R}^{N} \times \mathbb{R}^{N}=\left(\mathcal{S}_{\lambda} \cup \mathcal{S}_{\lambda}^{c} \cup \mathcal{D}_{\lambda} \cup \mathcal{D}_{\lambda}^{c}\right) \times\left(\mathcal{S}_{\lambda} \cup \mathcal{S}_{\lambda}^{c} \cup \mathcal{D}_{\lambda} \cup \mathcal{D}_{\lambda}^{c}\right)
$$

where $\mathcal{S}_{\lambda}, \mathcal{S}_{\lambda}^{c}, \mathcal{D}_{\lambda}$ and $\mathcal{D}_{\lambda}^{c}$ have been introduced in (3.2). By construction, it follows that

$$
\begin{aligned}
& \mathcal{G}(x, y)=\left[-\left(u(x)-u_{\lambda}(x)\right) w_{\lambda}(y) \varphi_{\varepsilon}^{2}(y)\right] \text { in }\left(\mathcal{S}_{\lambda}^{c} \times \mathcal{S}_{\lambda}\right), \\
& \mathcal{G}(x, y)=\left[-\left(u(x)-u_{\lambda}(x)\right) w_{\lambda}(y) \varphi_{\varepsilon}^{2}(y)\right] \text { in }\left(\mathcal{S}_{\lambda}^{c} \times \mathcal{D}_{\lambda}\right), \\
& \mathcal{G}(x, y)=\left[-\left(u(y)-u_{\lambda}(y)\right) w_{\lambda}(x) \varphi_{\varepsilon}^{2}(x)\right] \text { in }\left(\mathcal{S}_{\lambda} \times \mathcal{S}_{\lambda}^{c}\right), \\
& \mathcal{G}(x, y)=\left[-\left(u(y)-u_{\lambda}(y)\right) w_{\lambda}(x) \varphi_{\varepsilon}^{2}(x)\right] \text { in }\left(\mathcal{S}_{\lambda} \times \mathcal{D}_{\lambda}^{c}\right), \\
& \mathcal{G}(x, y)=\left[-\left(u(x)-u_{\lambda}(x)\right) w_{\lambda}(y) \varphi_{\varepsilon}^{2}(y)\right] \text { in }\left(\mathcal{D}_{\lambda}^{c} \times \mathcal{S}_{\lambda}\right),
\end{aligned}
$$




$$
\begin{array}{lc}
\mathcal{G}(x, y)=\left[-\left(u(x)-u_{\lambda}(x)\right) w_{\lambda}(y) \varphi_{\varepsilon}^{2}(y)\right] & \text { in }\left(\mathcal{D}_{\lambda}^{c} \times \mathcal{D}_{\lambda}\right), \\
\mathcal{G}(x, y)=\left[-\left(u(y)-u_{\lambda}(y)\right) w_{\lambda}(x) \varphi_{\varepsilon}^{2}(x)\right] \text { in }\left(\mathcal{D}_{\lambda} \times \mathcal{S}_{\lambda}^{c}\right), \\
\mathcal{G}(x, y)=\left[-\left(u(y)-u_{\lambda}(y)\right) w_{\lambda}(x) \varphi_{\varepsilon}^{2}(x)\right] \text { in }\left(\mathcal{D}_{\lambda} \times \mathcal{D}_{\lambda}^{c}\right) \\
\text { and } \mathcal{G}(x, y)=0 & \text { elsewhere } .
\end{array}
$$

We have that

$$
\int_{\mathcal{S}_{\lambda}^{c}} \int_{\mathcal{S}_{\lambda}} \frac{\mathcal{G}(x, y)}{|x-y|^{N+2 s}} \mathrm{~d} x \mathrm{~d} y+\int_{\mathcal{S}_{\lambda}^{c}} \int_{\mathcal{D}_{\lambda}} \frac{\mathcal{G}(x, y)}{|x-y|^{N+2 s}} \mathrm{~d} x \mathrm{~d} y \geq 0 .
$$

Indeed, note that, if $x \in \mathcal{S}_{\lambda}^{c}$ and $y \in \mathcal{S}_{\lambda}$, then $\mathcal{G}(x, y) \geq 0$; moreover, $\mathcal{G}(x, y)=-\mathcal{G}\left(x, y_{\lambda}\right)$. Also, we have that $|x-y| \leq\left|x-y_{\lambda}\right|$ for all $(x, y) \in \mathcal{S}_{\lambda}^{c} \times \mathcal{S}_{\lambda}$. Therefore, using also (3.3), we have

$$
\begin{aligned}
& \int_{\mathcal{S}_{\lambda}^{c}} \int_{\mathcal{S}_{\lambda}} \frac{\mathcal{G}(x, y)}{|x-y|^{N+2 s}} \mathrm{~d} x \mathrm{~d} y+\int_{\mathcal{S}_{\lambda}^{c}} \int_{\mathcal{D}_{\lambda}} \frac{\mathcal{G}(x, y)}{|x-y|^{N+2 s}} \mathrm{~d} x \mathrm{~d} y \\
& =\int_{\mathcal{S}_{\lambda}^{c}} \int_{\mathcal{S}_{\lambda}} \frac{\mathcal{G}(x, y)}{|x-y|^{N+2 s}} \mathrm{~d} x \mathrm{~d} y+\int_{\mathcal{S}_{\lambda}^{c}} \int_{\mathcal{S}_{\lambda}} \frac{\mathcal{G}\left(x, y_{\lambda}\right)}{\left|x-y_{\lambda}\right|^{N+2 s}} \mathrm{~d} x \mathrm{~d} y \\
& =\int_{\mathcal{S}_{\lambda}^{c}} \int_{\mathcal{S}_{\lambda}} \mathcal{G}(x, y)\left[\frac{1}{|x-y|^{N+2 s}}-\frac{1}{\left|x-y_{\lambda}\right|^{N+2 s}}\right] \mathrm{d} x \mathrm{~d} y \geq 0
\end{aligned}
$$

which shows (3.12). Similarly, one can prove that

$$
\begin{aligned}
& \int_{\mathcal{S}_{\lambda}} \int_{\mathcal{S}_{\lambda}^{c}} \frac{\mathcal{G}(x, y)}{|x-y|^{N+2 s}} \mathrm{~d} x \mathrm{~d} y+\int_{\mathcal{S}_{\lambda}} \int_{\mathcal{D}_{\lambda}^{c}} \frac{\mathcal{G}(x, y)}{|x-y|^{N+2 s}} \mathrm{~d} x \mathrm{~d} y \geq 0, \\
& \int_{\mathcal{D}_{\lambda}^{c}} \int_{\mathcal{S}_{\lambda}} \frac{\mathcal{G}(x, y)}{|x-y|^{N+2 s}} \mathrm{~d} x \mathrm{~d} y+\int_{\mathcal{D}_{\lambda}^{c}} \int_{\mathcal{D}_{\lambda}} \frac{\mathcal{G}(x, y)}{|x-y|^{N+2 s}} \mathrm{~d} x \mathrm{~d} y \geq 0
\end{aligned}
$$

and

$$
\int_{\mathcal{D}_{\lambda}} \int_{\mathcal{S}_{\lambda}^{c}} \frac{\mathcal{G}(x, y)}{|x-y|^{N+2 s}} \mathrm{~d} x \mathrm{~d} y+\int_{\mathcal{D}_{\lambda}} \int_{\mathcal{D}_{\lambda}^{c}} \frac{\mathcal{G}(x, y)}{|x-y|^{N+2 s}} \mathrm{~d} x \mathrm{~d} y \geq 0 .
$$

Collecting the estimates above we obtain (3.11) that actually proves (3.9) and the claim.

By (3.9) it follows now that (3.8) provides

$$
\begin{aligned}
& \frac{1}{2} c_{N, s} \int_{\mathbb{R}^{N}} \int_{\mathbb{R}^{N}} \frac{\left(w_{\lambda}(x)-w_{\lambda}(y)\right)\left(w_{\lambda}(x) \varphi_{\varepsilon}^{2}(x)-w_{\lambda}(y) \varphi_{\varepsilon}^{2}(y)\right)}{|x-y|^{N+2 s}} \mathrm{~d} x \mathrm{~d} y \\
& \quad \leq \int_{\Omega_{\lambda}}\left(\frac{f(x, u)-f\left(x, u_{\lambda}\right)}{u-u_{\lambda}}\right) w_{\lambda}^{2} \varphi_{\varepsilon}^{2} \mathrm{~d} x
\end{aligned}
$$

that we rewrite as

$$
\begin{aligned}
& \frac{1}{2} c_{N, s} \int_{\mathbb{R}^{N}} \int_{\mathbb{R}^{N}} \frac{\left(w_{\lambda}(x)-w_{\lambda}(y)\right)^{2} \varphi_{\varepsilon}^{2}(x)}{|x-y|^{N+2 s}} \mathrm{~d} x \mathrm{~d} y \\
& \leq \frac{1}{2} c_{N, s} \int_{\mathbb{R}^{N}} \int_{\mathbb{R}^{N}} \frac{\left(w_{\lambda}(x)-w_{\lambda}(y)\right)\left(\varphi_{\varepsilon}^{2}(y)-\varphi_{\varepsilon}^{2}(x)\right) w_{\lambda}(y)}{|x-y|^{N+2 s}} \mathrm{~d} x \mathrm{~d} y \\
& \quad+\int_{\Omega_{\lambda}}\left(\frac{f(x, u)-f\left(x, u_{\lambda}\right)}{u-u_{\lambda}}\right) w_{\lambda}^{2} \varphi_{\varepsilon}^{2} \mathrm{~d} x
\end{aligned}
$$




$$
\begin{aligned}
\leq & \frac{1}{2} c_{N, s} \int_{\mathbb{R}^{N}} \int_{\mathbb{R}^{N}} \frac{\left(w_{\lambda}(x)-w_{\lambda}(y)\right)\left(\varphi_{\varepsilon}^{2}(y)-\varphi_{\varepsilon}^{2}(x)\right) w_{\lambda}(y)}{|x-y|^{N+2 s}} \mathrm{~d} x \mathrm{~d} y \\
& +C\left(f, N, s,\|u\|_{L^{\infty}\left(\Omega_{\lambda}\right)}\right) \int_{\Omega_{\lambda}}\left(w_{\lambda}\right)^{2} \varphi_{\varepsilon}^{2} \mathrm{~d} x .
\end{aligned}
$$

Observe now that, by a symmetry argument, we have

$$
\begin{aligned}
& \int_{\mathbb{R}^{N}} \int_{\mathbb{R}^{N}} \frac{\left(w_{\lambda}(x)-w_{\lambda}(y)\right)^{2}}{|x-y|^{N+2 s}} \varphi_{\varepsilon}^{2}(x) \mathrm{d} x \mathrm{~d} y \\
& =\int_{\mathbb{R}^{N}} \int_{\mathbb{R}^{N}} \frac{\left(w_{\lambda}(x)-w_{\lambda}(y)\right)^{2}}{|x-y|^{N+2 s}}\left(\frac{\varphi_{\varepsilon}^{2}(x)+\varphi_{\varepsilon}^{2}(y)}{2}+\frac{\varphi_{\varepsilon}^{2}(x)-\varphi_{\varepsilon}^{2}(y)}{2}\right) \mathrm{d} x \mathrm{~d} y \\
& =\int_{\mathbb{R}^{N}} \int_{\mathbb{R}^{N}} \frac{\left(w_{\lambda}(x)-w_{\lambda}(y)\right)^{2}}{|x-y|^{N+2 s}}\left(\frac{\varphi_{\varepsilon}^{2}(x)+\varphi_{\varepsilon}^{2}(y)}{2}\right) \mathrm{d} x \mathrm{~d} y .
\end{aligned}
$$

On the other hand, using the Young inequality we have

$$
\begin{aligned}
& \left|\int_{\mathbb{R}^{N}} \int_{\mathbb{R}^{N}} \frac{\left(w_{\lambda}(x)-w_{\lambda}(y)\right)\left(\varphi_{\varepsilon}^{2}(y)-\varphi_{\varepsilon}^{2}(x)\right) w_{\lambda}(y)}{|x-y|^{N+2 s}} \mathrm{~d} x \mathrm{~d} y\right| \\
& =\left|\int_{\mathbb{R}^{N}} \int_{\mathbb{R}^{N}} \frac{\left(w_{\lambda}(x)-w_{\lambda}(y)\right) w_{\lambda}(y)}{|x-y|^{N+2 s}}\left(\varphi_{\varepsilon}(y)-\varphi_{\varepsilon}(x)\right)\left(\varphi_{\varepsilon}(x)+\varphi_{\varepsilon}(y)\right)\right| \\
& \leq \varepsilon \int_{\mathbb{R}^{N}} \int_{\mathbb{R}^{N}} \frac{\left(w_{\lambda}(x)-w_{\lambda}(y)\right)^{2}}{|x-y|^{N+2 s}}\left(\varphi_{\varepsilon}(x)+\varphi_{\varepsilon}(y)\right)^{2} \\
& \quad+\frac{C\left(f, N, s,\|u\|_{L^{\infty}\left(\Omega_{\lambda}\right)}\right)}{\varepsilon} \int_{\mathbb{R}^{N}} \int_{\mathbb{R}^{N}} \frac{\left(\varphi_{\varepsilon}(x)-\varphi_{\varepsilon}(y)\right)^{2}}{|x-y|^{N+2 s}} \\
& \leq 2 \varepsilon \int_{\mathbb{R}^{N}} \int_{\mathbb{R}^{N}} \frac{\left(w_{\lambda}(x)-w_{\lambda}(y)\right)^{2}}{|x-y|^{N+2 s}}\left(\varphi_{\varepsilon}^{2}(x)+\varphi_{\varepsilon}^{2}(y)\right) \\
& \quad+\frac{C\left(f, N, s,\|u\|_{L^{\infty}\left(\Omega_{\lambda}\right)}\right)}{\varepsilon} \int_{\mathbb{R}^{N}} \int_{\mathbb{R}^{N}} \frac{\left(\varphi_{\varepsilon}(x)-\varphi_{\varepsilon}(y)\right)^{2}}{|x-y|^{N+2 s}}
\end{aligned}
$$

In the following computations we set $\varepsilon=\frac{1}{8} c_{N, s}$ and, taking into account (3.14), by (3.15) and (3.16), we arrive at

$$
\begin{aligned}
& \int_{\mathbb{R}^{N}} \int_{\mathbb{R}^{N}} \frac{\left(w_{\lambda}(x)-w_{\lambda}(y)\right)^{2}}{|x-y|^{N+2 s}}\left(\frac{\varphi_{\varepsilon}^{2}(x)+\varphi_{\varepsilon}^{2}(y)}{2}\right) \mathrm{d} x \mathrm{~d} y \\
& \leq C\left(f, N, s,\|u\|_{L^{\infty}\left(\Omega_{\lambda}\right)}\right) \int_{\Omega_{\lambda}} w_{\lambda}^{2} \varphi_{\varepsilon}^{2} \mathrm{~d} x \\
& \quad+C\left(f, N, s,\|u\|_{L^{\infty}\left(\Omega_{\lambda}\right)}\right) \int_{\mathbb{R}^{N}} \int_{\mathbb{R}^{N}} \frac{\left(\varphi_{\varepsilon}(x)-\varphi_{\varepsilon}(y)\right)^{2}}{|x-y|^{N+2 s}} \\
& \leq C\left(f, N, s,\|u\|_{L^{\infty}\left(\Omega_{\lambda}\right)}\right) .
\end{aligned}
$$

In the final estimate we exploited the properties of the cutoff function provided by (3.6) and the fact that $0 \leq w_{\lambda} \leq u$ in $\Omega_{\lambda}$ (together with a symmetry argument).

Then, since $\varphi_{\varepsilon} \rightarrow 1$ in $\mathbb{R}^{N}$ as $\varepsilon \rightarrow 0^{+}$, the inequality (3.4) follows by Fatou Lemma letting $\varepsilon \rightarrow 0^{+}$in (3.17).

To deduce that $w_{\lambda} \in \mathcal{H}_{0}^{s}\left(\Omega_{\lambda} \cup R_{\lambda}\left(\Omega_{\lambda}\right)\right)$ just note that $w_{\lambda}$ is bounded and then apply standard arguments, see [9]. 
Proof of Theorem 1.1 We start the moving plane procedure by showing that, recalling (2.24), we can take $a<\lambda<0$, with $|\lambda-a|$ small, in such a way that $u \leq u_{\lambda}$ in $\Omega_{\lambda} \backslash R_{\lambda}(\Gamma)$. In fact using $\varphi:=w_{\lambda} \varphi_{\varepsilon}^{2}$ in Eq. (1.1) fulfilled by $u$ and in Eq. (3.7) fulfilled by $u_{\lambda}$, subtracting we get

$$
\begin{aligned}
& \frac{1}{2} c_{N, s} \int_{\mathbb{R}^{N}} \int_{\mathbb{R}^{N}} \frac{\left(\left(u(x)-u_{\lambda}(x)\right)-\left(u(y)-u_{\lambda}(y)\right)\right)}{|x-y|^{N+2 s}}\left(w_{\lambda}(x) \varphi_{\varepsilon}^{2}(x)-w_{\lambda}(y) \varphi_{\varepsilon}^{2}(y)\right) \mathrm{d} x \mathrm{~d} y \\
& \quad \leq \int_{\mathbb{R}^{N}}\left(\frac{f(x, u)-f\left(x, u_{\lambda}\right)}{u-u_{\lambda}}\right) w_{\lambda}^{2} \varphi_{\varepsilon}^{2} \mathrm{~d} x
\end{aligned}
$$

and then, as in (3.13) (see also (3.9)), we have

$$
\begin{aligned}
& \frac{1}{2} c_{N, s} \int_{\mathbb{R}^{N}} \int_{\mathbb{R}^{N}} \frac{\left(w_{\lambda}(x)-w_{\lambda}(y)\right)\left(w_{\lambda}(x) \varphi_{\varepsilon}^{2}(x)-w_{\lambda}(y) \varphi_{\varepsilon}^{2}(y)\right)}{|x-y|^{N+2 s}} \mathrm{~d} x \mathrm{~d} y \\
& \quad \leq \int_{\mathbb{R}^{N}}\left(\frac{f(x, u)-f\left(x, u_{\lambda}\right)}{u-u_{\lambda}}\right) w_{\lambda}^{2} \varphi_{\varepsilon}^{2} \mathrm{~d} x .
\end{aligned}
$$

Using that $\varphi_{\varepsilon}^{2} \leq 1$ in all $\mathbb{R}^{N}$ and that $w \in L^{\infty}\left(\mathbb{R}^{N}\right)$, it follows

$$
\begin{aligned}
& \int_{\mathbb{R}^{N}} \int_{\mathbb{R}^{N}} \frac{\left(w_{\lambda}(x) \varphi_{\varepsilon}^{2}(x)-w_{\lambda}(y) \varphi_{\varepsilon}^{2}(y)\right)^{2}}{|x-y|^{N+2 s}} \mathrm{~d} x \mathrm{~d} y \\
& \leq 2 \int_{\mathbb{R}^{N}} \int_{\mathbb{R}^{N}} \frac{\left(w_{\lambda}(x)-w_{\lambda}(y)\right)^{2}}{|x-y|^{N+2 s}} \mathrm{~d} x \mathrm{~d} y \\
& \quad+C \int_{\mathbb{R}^{N}} \int_{\mathbb{R}^{N}} \frac{\left(\varphi_{\varepsilon}(y)-\varphi_{\varepsilon}(x)\right)^{2}}{|x-y|^{N+2 s}} \mathrm{~d} x \mathrm{~d} y
\end{aligned}
$$

and $C=C\left(\|u\|_{L^{\infty}\left(\Omega_{\lambda}\right)}\right)$ is a positive constant. Therefore, by Lemma 3.1, (3.5) and (3.6) we deduce

$$
\int_{\mathbb{R}^{N}} \int_{\mathbb{R}^{N}} \frac{\left(w_{\lambda}(x) \varphi_{\varepsilon}^{2}(x)-w_{\lambda}(y) \varphi_{\varepsilon}^{2}(y)\right)^{2}}{|x-y|^{N+2 s}} \mathrm{~d} x \mathrm{~d} y \leq C,
$$

where $C$ is a positive constant not depending on $\varepsilon$. Letting $\varepsilon$ tend to zero, the 1.h.s of (3.18) by weak convergence goes to

$$
\frac{1}{2} c_{N, s} \int_{\mathbb{R}^{N}} \int_{\mathbb{R}^{N}} \frac{\left(w_{\lambda}(x)-w_{\lambda}(y)\right)^{2}}{|x-y|^{N+2 s}} \mathrm{~d} x \mathrm{~d} y .
$$

By $\left(A_{f}^{1}\right)$ and Lemma 3.1, the r.h.s of (3.18) goes to

$$
\int_{\mathbb{R}^{N}}\left(\frac{f(x, u)-f\left(x, u_{\lambda}\right)}{u-u_{\lambda}}\right) w_{\lambda}^{2} \mathrm{~d} x .
$$

Hence, (3.18) becomes

$$
\frac{1}{2} c_{N, s} \int_{\mathbb{R}^{N}} \int_{\mathbb{R}^{N}} \frac{\left(w_{\lambda}(x)-w_{\lambda}(y)\right)^{2}}{|x-y|^{N+2 s}} \mathrm{~d} x \mathrm{~d} y \leq \int_{\mathbb{R}^{N}}\left(\frac{f(x, u)-f\left(x, u_{\lambda}\right)}{u-u_{\lambda}}\right) w_{\lambda}^{2} \mathrm{~d} x .
$$

Using $\left(A_{f}^{1}\right)$ and Hölder inequality, it follows

$$
\frac{1}{2} c_{N, s} \int_{\mathbb{R}^{N}} \int_{\mathbb{R}^{N}} \frac{\left(w_{\lambda}(x)-w_{\lambda}(y)\right)^{2}}{|x-y|^{N+2 s}} \mathrm{~d} x \mathrm{~d} y
$$




$$
\begin{aligned}
& \leq \int_{\mathbb{R}^{N}}\left(\frac{f(x, u)-f\left(x, u_{\lambda}\right)}{u-u_{\lambda}}\right) w_{\lambda}^{2} \mathrm{~d} x \\
& \leq 2 C_{f}\left|\Omega_{\lambda}\right|^{\frac{2_{s}^{*}-2}{2_{s}^{*}}}\left(\int_{\Sigma_{\lambda}} w_{\lambda}^{2_{s}^{*}} \mathrm{~d} x\right)^{\frac{2}{2_{s}^{*}}} \\
& \leq \frac{4 C_{f}}{S_{N, s} c_{N, s}}\left|\Omega_{\lambda}\right|^{\frac{2_{s}^{*}-2}{2_{s}^{*}}} \int_{\mathbb{R}^{N}} \int_{\mathbb{R}^{N}} \frac{\left(w_{\lambda}(x)-w_{\lambda}(y)\right)^{2}}{|x-y|^{N+2 s}} \mathrm{~d} x \mathrm{~d} y,
\end{aligned}
$$

where the last inequality follows from Theorem 2.1. Recalling (2.24), for $|\lambda-a|$ small, it follows that

$$
\frac{4 C_{f}}{S_{N, s} c_{N, s}}\left|\Omega_{\lambda}\right|^{\frac{2_{s}^{*}-2}{2_{s}^{*}}}<\frac{1}{4} c_{N, s} .
$$

A contradiction occurs by (3.20) unless

$$
\int_{\mathbb{R}^{N}} \int_{\mathbb{R}^{N}} \frac{\left(w_{\lambda}(x)-w_{\lambda}(y)\right)^{2}}{|x-y|^{N+2 s}} \mathrm{~d} x \mathrm{~d} y=0,
$$

that is $u \leq u_{\lambda}$ in $\Omega_{\lambda}$.

Let us now set

$$
\Lambda_{0}=\left\{a<\lambda<0: u \leq u_{t} \text { in } \Omega_{t} \backslash R_{t}(\Gamma) \text { for all } t \in(a, \lambda]\right\}
$$

and

$$
\lambda_{0}=\sup \Lambda_{0} .
$$

that is well defined since we showed that $\Lambda_{0}$ is not empty. To prove our result we have to show that $\lambda_{0}=0$.

To prove this we assume that $\lambda_{0}<0$ and we reach a contradiction by proving that $u \leq u_{\lambda_{0}+\tau}$ in $\Omega_{\lambda_{0}+\tau} \backslash R_{\lambda_{0}+\tau}(\Gamma)$ for any $0<\tau<\bar{\tau}$ for some small $\bar{\tau}>0$. By continuity of $u$ in $\bar{\Omega} \backslash \Gamma$, we know that $u \leq u_{\lambda_{0}}$ in $\Omega_{\lambda_{0}} \backslash R_{\lambda_{0}}(\Gamma)$. Actually it follows that $u<u_{\lambda_{0}}$ in $\Omega_{\lambda_{0}} \backslash R_{\lambda_{0}}(\Gamma)$. To deduce this, just write down the equation fulfilled by $u-u_{\lambda_{0}}$ and exploit Proposition 3.6 in [17].

Therefore, given a compact set $K \subset \Omega_{\lambda_{0}} \backslash \overline{R_{\lambda_{0}}(\Gamma)}$, by a uniform continuity argument, we can ensure that $u<u_{\lambda_{0}+\tau}$ in $K$ for any $0<\tau<\bar{\tau}$ for $\bar{\tau}>0$ small. Note that to do this we implicitly assume, with no loss of generality, that $R_{\lambda_{0}+\tau}(\Gamma)$ remains bounded away from $K$. Arguing as in Lemma 3.1 we consider

$$
\varphi_{\varepsilon}=\varphi_{\varepsilon}^{\lambda_{0}+\tau}, \quad 0<\tau<\bar{\tau}
$$

with the same construction and we set

$$
\varphi:=w_{\lambda_{0}+\tau} \varphi_{\varepsilon}^{2}
$$

In view of Lemma 3.1, we can choose $\varphi$ as test function arguing exactly as in the proof of Lemma 3.1 and again we arrive at the first inequality in (3.17), namely

$$
\begin{aligned}
& \int_{\mathbb{R}^{N}} \int_{\mathbb{R}^{N}} \frac{\left(w_{\lambda_{0}+\tau}(x)-w_{\lambda_{0}+\tau}(y)\right)^{2}}{|x-y|^{N+2 s}}\left(\frac{\varphi_{\varepsilon}^{2}(x)+\varphi_{\varepsilon}^{2}(y)}{2}\right) \mathrm{d} x \mathrm{~d} y \\
& \quad \leq C\left(f, N, s,\|u\|_{L^{\infty}\left(\Omega_{\lambda_{0}+\bar{\tau}}\right)}\right) \int_{\Omega_{\lambda_{0}+\tau}}\left(w_{\lambda_{0}+\tau}\right)^{2} \varphi_{\varepsilon}^{2} \mathrm{~d} x
\end{aligned}
$$




$$
+C\left(f, N, s,\|u\|_{L^{\infty}\left(\Omega_{\lambda_{0}+\bar{\tau}}\right)}\right) \int_{\mathbb{R}^{N}} \int_{\mathbb{R}^{N}} \frac{\left(\varphi_{\varepsilon}(x)-\varphi_{\varepsilon}(y)\right)^{2}}{|x-y|^{N+2 s}} .
$$

By construction, see (3.6), it follows that

$$
\int_{\mathbb{R}^{N}} \int_{\mathbb{R}^{N}} \frac{\left(\varphi_{\varepsilon}(x)-\varphi_{\varepsilon}(y)\right)^{2}}{|x-y|^{N+2 s}} \underset{\varepsilon \rightarrow 0}{\longrightarrow} 0 .
$$

Therefore, arguing as above, we pass to the limit as $\varepsilon \rightarrow 0$ and, recalling Lemma 3.1, we deduce that

$$
\begin{aligned}
& \int_{\mathbb{R}^{N}} \int_{\mathbb{R}^{N}} \frac{\left(w_{\lambda_{0}+\tau}(x)-w_{\lambda_{0}+\tau}(y)\right)^{2}}{|x-y|^{N+2 s}} \mathrm{~d} x \mathrm{~d} y . \\
& \quad \leq C\left(f, N, s,\|u\|_{L^{\infty}\left(\Omega_{\lambda_{0}+\bar{\tau}}\right)}\right) \int_{\Omega_{\lambda_{0}+\tau}}\left(w_{\lambda_{0}+\tau}\right)^{2} \mathrm{~d} x \\
& \quad=C\left(f, N, s,\|u\|_{L^{\infty}\left(\Omega_{\lambda_{0}+\bar{\tau}}\right)}\right) \int_{\Omega_{\lambda_{0}+\tau} \backslash K}\left(w_{\lambda_{0}+\tau}\right)^{2} \mathrm{~d} x .
\end{aligned}
$$

By the Sobolev inequality, see Theorem 2.1, we deduce that

$$
\begin{aligned}
& \int_{\mathbb{R}^{N}} \int_{\mathbb{R}^{N}} \frac{\left(w_{\lambda_{0}+\tau}(x)-w_{\lambda_{0}+\tau}(y)\right)^{2}}{|x-y|^{N+2 s}} \mathrm{~d} x \mathrm{~d} y \\
& \leq C\left(f, N, s,\|u\|_{L^{\infty}\left(\Omega_{\lambda_{0}+\bar{\tau}}\right)}\right)\left|\left(\Omega_{\lambda_{0}+\tau} \backslash K\right)\right|^{\frac{2_{s}^{*}-2}{2_{s}^{*}}}\left(\int_{\Omega_{\lambda_{0}+\tau} \backslash K}\left(w_{\lambda_{0}+\tau}\right)^{2_{s}^{*}} \mathrm{~d} x\right)^{\frac{2}{2_{s}^{*}}} \\
& \leq C\left(f, N, s,\|u\|_{L^{\infty}\left(\Omega_{\lambda_{0}+\bar{\tau}}\right)}\right)\left|\left(\Omega_{\lambda_{0}+\tau} \backslash K\right)\right|^{\frac{2_{s}^{*}-2}{2_{s}^{*}}} \int_{\mathbb{R}^{N}} \int_{\mathbb{R}^{N}} \frac{\left(w_{\lambda_{0}+\tau}(x)-w_{\lambda_{0}+\tau}(y)\right)^{2}}{|x-y|^{N+2 s}} \mathrm{~d} x \mathrm{~d} y
\end{aligned}
$$

where the $C(\cdot)$ involves now the Sobolev constant. For $K$ large and $\bar{\tau}$ small, we may assume that

$$
c\left(f, N, s,\|u\|_{L^{\infty}\left(\Omega_{\lambda_{0}+\bar{\tau}}\right)}\right)\left|\left(\Omega_{\lambda_{0}+\tau} \backslash K\right)\right|^{\frac{2_{s}^{*}-2}{2_{s}^{*}}}<1
$$

so that, by (3.21), we deduce that

$$
\int_{\mathbb{R}^{N}} \int_{\mathbb{R}^{N}} \frac{\left(w_{\lambda_{0}+\tau}(x)-w_{\lambda_{0}+\tau}(y)\right)^{2}}{|x-y|^{N+2 s}} \mathrm{~d} x \mathrm{~d} y=0 .
$$

This proves that $u \leq u_{\lambda_{0}+\tau}$ in $\Omega_{\lambda_{0}+\tau} \backslash R_{\lambda_{0}+\tau}(\Gamma)$ for any $0<\tau<\bar{\tau}$ and for some small $\bar{\tau}>0$. Such a contradiction shows that

$$
\lambda_{0}=0
$$

Since the moving plane procedure can be performed in the same way but in the opposite direction, then this proves the desired symmetry result. The fact that the solution is increasing in the $x_{1}$-direction in $\left\{x_{1}<0\right\}$ is implicit in the moving plane procedure. If $\Omega$ is a ball and $u$ has only a nonremovable singularity at the origin, then the solution is radial and radially decreasing about the center of the ball. This follows applying the moving plane procedure in any direction $v \in \mathbb{S}^{1}$ of $\mathbb{R}^{N}$. 


\section{Proof of Theorem 1.2}

We start by proving the following

Lemma 4.1 Under the assumptions of Theorem 1.2, for $\lambda<0$, we have that

$$
\int_{\mathbb{R}^{N}} \int_{\mathbb{R}^{N}} \frac{\left(w_{\lambda}(x)-w_{\lambda}(y)\right)^{2}}{|x-y|^{N+2 s}} \mathrm{~d} x \mathrm{~d} y \leq C,
$$

where $C=C\left(f, s, N,\|u\|_{L^{2 *}\left(\mathbb{R}^{N} \backslash B_{R_{0}}\right)},\|u\|_{L^{\infty}\left(\Sigma_{\lambda} \cap B_{R_{0}}\right)}\right)$ is a positive constant.

Proof We start by exploiting the fact that the singular set $\Gamma$ has zero $s$-capacity. For each $\varepsilon>0$, let

$$
\Gamma_{\varepsilon}^{\lambda}:=\left\{x \in \mathbb{R}^{N} \mid \operatorname{dist}\left(x, R_{\lambda}(\Gamma)\right)<\varepsilon\right\} .
$$

Arguing as in the case of a bounded domain, thanks to Lemma 2.5, we have that, for each $\varepsilon>0, \operatorname{Cap}_{s}^{\Gamma_{\varepsilon}^{\lambda}}\left(R_{\lambda}(\Gamma)\right)=0$. Therefore, there exists $\phi_{\varepsilon} \in C_{c}^{\infty}\left(\Gamma_{\varepsilon}^{\lambda}\right)$ such that

$$
\int_{\mathbb{R}^{N}} \int_{\mathbb{R}^{N}} \frac{\left(\phi_{\varepsilon}(x)-\phi_{\varepsilon}(y)\right)^{2}}{|x-y|^{N+2 s}} \mathrm{~d} x \mathrm{~d} y \leq \varepsilon,
$$

with $\phi_{\varepsilon} \geq 1$ on a neighborhood of $R_{\lambda}(\Gamma)$. Via a truncation argument it follows that we can assume $0 \leq \phi_{\varepsilon} \leq 1, \phi_{\varepsilon} \in H_{0}^{s}\left(\Gamma_{\varepsilon}^{\lambda}\right)$. Let $\varphi_{\varepsilon}^{\lambda}(x)$ be defined in $\Sigma_{\lambda}$ as in (3.6). Then, by even reflection, we define $\varphi_{\varepsilon}^{\lambda}(x)$ in all $\mathbb{R}^{N}$ putting $\varphi_{\varepsilon}^{\lambda}(x)=\varphi_{\varepsilon}^{\lambda}\left(x_{\lambda}\right)$ for every $x \in \mathbb{R}^{n} \backslash \Sigma_{\lambda}$. Let $\varphi_{1,0} \in C^{\infty}\left(\mathbb{R}^{N}\right)$ be a standard cutoff function such that $\varphi_{1,0}=1$ in $B_{1}(0)$ and $\varphi_{1,0}=0$ outside $B_{2}(0)$ and even w.r.t the hyperplane $T_{0}$, i.e., $\varphi_{1,0}(x)=\varphi_{1,0}\left(x_{0}\right)$ for every $x \in \mathbb{R}^{n} \backslash \Sigma_{0}$. Then, for a fixed point $x_{C} \in T_{\lambda}$, let us set $\varphi_{R, x_{c}}=\varphi_{1,0}\left(\left(x-x_{C}\right) / R\right)$. Recalling (3.1) we set

$$
\varphi:=w_{\lambda} \varphi_{\varepsilon}^{2} \varphi_{R, x_{c}}^{2} .
$$

We point out that $u_{\lambda}$ (see (2.23)) solves

$$
(-\Delta)^{s} u_{\lambda}=f\left(u_{\lambda}\right) \text { in } \mathbb{R}^{N} \backslash R_{\lambda}(\Gamma),
$$

in the sense of Definition 2.2. By density arguments (see Lemma 2.4), we can plug $\varphi$ as test function in Eq. (1.2) fulfilled by $u$ and in equation (4.3) fulfilled by $u_{\lambda}$. Subtracting, we get

$$
\begin{aligned}
& \frac{1}{2} c_{N, s} \int_{\mathbb{R}^{N}} \int_{\mathbb{R}^{N}} \frac{\left(\left(u(x)-u_{\lambda}(x)\right)-\left(u(y)-u_{\lambda}(y)\right)\right)}{|x-y|^{N+2 s}} \\
& \quad \times\left(w_{\lambda}(x) \varphi_{\varepsilon}^{2}(x) \varphi_{R, x_{c}}^{2}(x)-w_{\lambda}(y) \varphi_{\varepsilon}^{2}(y) \varphi_{R, x_{c}}^{2}(y)\right) \mathrm{d} x \mathrm{~d} y \\
& \quad \leq \int_{\mathbb{R}^{N}}\left(\frac{f(u)-f\left(u_{\lambda}\right)}{u-u_{\lambda}}\right) w_{\lambda}^{2} \varphi_{\varepsilon}^{2} \varphi_{R, x_{c}}^{2} \mathrm{~d} x .
\end{aligned}
$$

Arguing as in the proof of Lemma 3.1, following verbatim the computations from Eq. (3.9) to equation (3.13), we obtain

$$
\begin{aligned}
& \frac{1}{2} c_{N, s} \int_{\mathbb{R}^{N}} \int_{\mathbb{R}^{N}} \frac{\left(w_{\lambda}(x)-w_{\lambda}(y)\right)\left(w_{\lambda}(x) \varphi_{\varepsilon}^{2}(x) \varphi_{R, x_{c}}^{2}(x)-w_{\lambda}(y) \varphi_{\varepsilon}^{2}(y) \varphi_{R, x_{c}}^{2}(y)\right)}{|x-y|^{N+2 s}} \mathrm{~d} x \mathrm{~d} y \\
& \quad \leq \int_{\mathbb{R}^{N}}\left(\frac{f(u)-f\left(u_{\lambda}\right)}{u-u_{\lambda}}\right) w_{\lambda}^{2} \varphi_{\varepsilon}^{2} \varphi_{R, x_{c}}^{2} \mathrm{~d} x .
\end{aligned}
$$


We rewrite (4.5) as

$$
\begin{aligned}
& \frac{1}{2} c_{N, s} \int_{\mathbb{R}^{N}} \int_{\mathbb{R}^{N}} \frac{\left(w_{\lambda}(x)-w_{\lambda}(y)\right)^{2} \varphi_{\varepsilon}^{2}(x) \varphi_{R, x_{c}}^{2}(x)}{|x-y|^{N+2 s}} \mathrm{~d} x \mathrm{~d} y \\
& \leq \frac{1}{2} c_{N, s} \int_{\mathbb{R}^{N}} \int_{\mathbb{R}^{N}} \frac{\left(w_{\lambda}(x)-w_{\lambda}(y)\right)\left(\varphi_{\varepsilon}^{2}(y) \varphi_{R, x_{c}}^{2}(y)-\varphi_{\varepsilon}^{2}(x) \varphi_{R, x_{c}}^{2}(x)\right) w_{\lambda}(y)}{|x-y|^{N+2 s}} \mathrm{~d} x \mathrm{~d} y \\
& \quad+\int_{\mathbb{R}^{N}}\left(\frac{f(u)-f\left(u_{\lambda}\right)}{u-u_{\lambda}}\right) w_{\lambda}^{2} \varphi_{\varepsilon}^{2} \varphi_{R, x_{c}}^{2} \mathrm{~d} x .
\end{aligned}
$$

Recalling (3.15) we have

$$
\begin{aligned}
& \int_{\mathbb{R}^{N}} \int_{\mathbb{R}^{N}} \frac{\left(w_{\lambda}(x)-w_{\lambda}(y)\right)^{2} \varphi_{\varepsilon}^{2}(x) \varphi_{R, x_{c}}^{2}(x)}{|x-y|^{N+2 s}} \mathrm{~d} x \mathrm{~d} y \\
& \quad=\int_{\mathbb{R}^{N}} \int_{\mathbb{R}^{N}} \frac{\left(w_{\lambda}(x)-w_{\lambda}(y)\right)^{2}}{|x-y|^{N+2 s}}\left(\frac{\varphi_{\varepsilon}^{2}(x) \varphi_{R, x_{c}}^{2}(x)+\varphi_{\varepsilon}^{2}(y) \varphi_{R, x_{c}}^{2}(y)}{2}\right) \mathrm{d} x \mathrm{~d} y .
\end{aligned}
$$

On the other hand, using the Young inequality we have

$$
\begin{aligned}
& \left|\int_{\mathbb{R}^{N}} \int_{\mathbb{R}^{N}} \frac{\left(w_{\lambda}(x)-w_{\lambda}(y)\right)\left(\varphi_{\varepsilon}^{2}(y) \varphi_{R, x_{c}}^{2}(y)-\varphi_{\varepsilon}^{2}(x) \varphi_{R, x_{c}}^{2}(x)\right) w_{\lambda}(y)}{|x-y|^{N+2 s}} \mathrm{~d} x \mathrm{~d} y\right| \\
& =\mid \int_{\mathbb{R}^{N}} \int_{\mathbb{R}^{N}} \frac{\left(w_{\lambda}(x)-w_{\lambda}(y)\right) w_{\lambda}(y)}{|x-y|^{N+2 s}} \\
& \quad \times\left(\varphi_{\varepsilon}(y) \varphi_{R, x_{c}}(y)-\varphi_{\varepsilon}(x) \varphi_{R, x_{c}}(x)\right)\left(\varphi_{\varepsilon}(x) \varphi_{R, x_{c}}(x)+\varphi_{\varepsilon}(y) \varphi_{R, x_{c}}(y)\right) \mathrm{d} x \mathrm{~d} y \mid \\
& \leq \\
& \quad \delta \int_{\mathbb{R}^{N}} \int_{\mathbb{R}^{N}} \frac{\left(w_{\lambda}(x)-w_{\lambda}(y)\right)^{2}}{|x-y|^{N+2 s}}\left(\varphi_{\varepsilon}(x) \varphi_{R, x_{c}}(x)+\varphi_{\varepsilon}(y) \varphi_{R, x_{c}}(y)\right)^{2} \mathrm{~d} x \mathrm{~d} y \\
& \quad+\frac{1}{\delta} \int_{\mathbb{R}^{N}} \int_{\mathbb{R}^{N}} \frac{\left(\varphi_{\varepsilon}(x) \varphi_{R, x_{c}}(x)-\varphi_{\varepsilon}(y) \varphi_{R, x_{c}}(y)\right)^{2} w^{2}(y)}{|x-y|^{N+2 s} \mathrm{~d} x \mathrm{~d} y} \\
& \leq 2 \delta \int_{\mathbb{R}^{N}} \int_{\mathbb{R}^{N}} \frac{\left(w_{\lambda}(x)-w_{\lambda}(y)\right)^{2}}{|x-y|^{N+2 s}}\left(\varphi_{\varepsilon}^{2}(x) \varphi_{R}^{2}(x)+\varphi_{\varepsilon}^{2}(y) \varphi_{R}^{2}(y)\right) \mathrm{d} x \mathrm{~d} y \\
& \quad+\frac{1}{\delta} \int_{\mathbb{R}^{N}} \int_{\mathbb{R}^{N}} \frac{\left(\varphi_{\varepsilon}(x) \varphi_{R, x_{c}}(x)-\varphi_{\varepsilon}(y) \varphi_{R, x_{c}}(y)\right)^{2} w^{2}(y)}{|x-y|^{N+2 s}} \mathrm{~d} x \mathrm{~d} y .
\end{aligned}
$$

Now we set $\delta=\frac{1}{8} c_{N, s}$ and, taking into account (4.6), by (4.7) and (4.8) we obtain

$$
\begin{aligned}
& \int_{\mathbb{R}^{N}} \int_{\mathbb{R}^{N}} \frac{\left(w_{\lambda}(x)-w_{\lambda}(y)\right)^{2}}{|x-y|^{N+2 s}}\left(\frac{\varphi_{\varepsilon}^{2}(x) \varphi_{R, x_{c}}^{2}(x)+\varphi_{\varepsilon}^{2}(y) \varphi_{R, x_{c}}^{2}(y)}{2}\right) \mathrm{d} x \mathrm{~d} y \\
& \leq C \int_{\mathbb{R}^{N}} \int_{\mathbb{R}^{N}} \frac{\left(\varphi_{\varepsilon}(x) \varphi_{R, x_{c}}(x)-\varphi_{\varepsilon}(y) \varphi_{R, x_{c}}(y)\right)^{2} w_{\lambda}^{2}(y)}{|x-y|^{N+2 s}} \mathrm{~d} x \mathrm{~d} y \\
& \quad+\int_{\mathbb{R}^{N}}\left(\frac{f(u)-f\left(u_{\lambda}\right)}{u-u_{\lambda}}\right) w_{\lambda}^{2} \varphi_{\varepsilon}^{2} \varphi_{R, x_{c}}^{2} \mathrm{~d} x \\
& \quad:=I_{1}+I_{2}
\end{aligned}
$$


where $C$ is a positive constant depending on $s, N$. Let us start by evaluating the term $I_{1}$. First of all we obtain

$$
\begin{aligned}
I_{1} \leq & C \int_{\mathbb{R}^{N}} \int_{\mathbb{R}^{N}} \frac{\left|\varphi_{R, x_{c}}(x)-\varphi_{R, x_{c}}(y)\right|^{2} \varphi_{\varepsilon}^{2}(y) w_{\lambda}^{2}(y)}{|x-y|^{N+2 s}} \mathrm{~d} x \mathrm{~d} y \\
& +C \int_{\mathbb{R}^{N}} \int_{\mathbb{R}^{N}} \frac{\left|\varphi_{\varepsilon}(x)-\varphi_{\varepsilon}(y)\right|^{2} \varphi_{R}^{2}(x) w_{\lambda}^{2}(y)}{|x-y|^{N+2 s}} \mathrm{~d} x \mathrm{~d} y \\
\leq & C \int_{\mathbb{R}^{N}} \int_{\mathbb{R}^{N}} \frac{\left|\varphi_{R, x_{c}}(x)-\varphi_{R, x_{c}}(y)\right|^{2} w_{\lambda}^{2}(y)}{|x-y|^{N+2 s}} \mathrm{~d} x \mathrm{~d} y \\
& +C \int_{\mathbb{R}^{N}} \int_{\mathbb{R}^{N}} \frac{\left|\varphi_{\varepsilon}(x)-\varphi_{\varepsilon}(y)\right|^{2} w_{\lambda}^{2}(y)}{|x-y|^{N+2 s}} \mathrm{~d} x \mathrm{~d} y \\
:= & I_{11}+I_{12} .
\end{aligned}
$$

where we also used that $\varphi_{R}^{2} \leq 1, \varphi_{\varepsilon}^{2} \leq 1$ in a $\mathbb{R}^{N}$. In the following we exploit some standard arguments, see, for example, [10]. In our case such an application would be more easy in the case of globally bounded solutions. Since we deal with the more general case of locally bounded solutions, the computations are more involved.

To estimate the term $I_{11}$, we define the following sets:

$$
\begin{aligned}
& A_{0}\left(x_{C}\right):=\left\{(x, y) \in \mathbb{R}^{N} \times \mathbb{R}^{N}:\left|y-x_{C}\right| \geq 2\left|x-x_{C}\right|\right\}, \\
& A_{1}\left(x_{C}\right):=\left\{(x, y) \in \mathbb{R}^{N} \times \mathbb{R}^{N}:\left|y-x_{C}\right|<2\left|x-x_{C}\right| \text { and }|x-y| \geq R\right\}, \\
& A_{2}\left(x_{C}\right):=\left\{(x, y) \in \mathbb{R}^{N} \times \mathbb{R}^{N}:\left|y-x_{C}\right|<2\left|x-x_{C}\right| \text { and }|x-y|<R\right\},
\end{aligned}
$$

Therefore,

$$
\begin{aligned}
I_{11}= & C \iint_{A_{0}\left(x_{C}\right)} \frac{\left|\varphi_{R, x_{c}}(x)-\varphi_{R, x_{c}}(y)\right|^{2} w_{\lambda}^{2}(y)}{|x-y|^{N+2 s}} \mathrm{~d} x \mathrm{~d} y \\
& +C \iint_{A_{1}\left(x_{C}\right)} \frac{\left|\varphi_{R, x_{c}}(x)-\varphi_{R, x_{c}}(y)\right|^{2} w_{\lambda}^{2}(y)}{|x-y|^{N+2 s}} \mathrm{~d} x \mathrm{~d} y \\
& +C \iint_{A_{2}\left(x_{C}\right)} \frac{\left|\varphi_{R, x_{c}}(x)-\varphi_{R, x_{c}}(y)\right|^{2} w_{\lambda}^{2}(y)}{|x-y|^{N+2 s}} \mathrm{~d} x \mathrm{~d} y \\
:= & \sum_{k=0}^{2} I_{11 k},
\end{aligned}
$$

Define $\sigma_{0}=s$ and fix $\sigma_{1} \in(0, s)$ and $\sigma_{2} \in(s, 1)$. Let us write now, for $k=0,1,2$,

$$
\frac{\left|\varphi_{R, x_{c}}(x)-\varphi_{R, x_{c}}(y)\right|^{2} w_{\lambda}^{2}(y)}{|x-y|^{N+2 s}}=\frac{\left|\varphi_{R, x_{c}}(x)-\varphi_{R, x_{c}}(y)\right|^{2}}{|x-y|^{2\left(s+\sigma_{k}\right)}} \frac{w_{\lambda}^{2}(y)}{|x-y|^{N-2 \sigma_{k}}} .
$$

By Hölder inequality, for $k=\{0,1,2\}$, we have

$$
I_{11 k} \leq C\left(\iint_{A_{k}\left(x_{C}\right)} \frac{\left|\varphi_{R, x_{C}}(x)-\varphi_{R, x_{C}}(y)\right|^{\frac{N}{s}}}{|x-y|^{\left(s+\sigma_{k}\right) \frac{N}{s}}} \mathrm{~d} x \mathrm{~d} y\right)^{\frac{2 s}{N}}\left(\iint_{A_{k}\left(x_{C}\right)} \frac{|w|_{\lambda}^{2^{*}}(y)}{|x-y|^{\frac{N-2 \sigma_{k}}{N-2 s}}} \mathrm{~d} x \mathrm{~d} y\right)^{\frac{N-2 s}{N}} .
$$


The first integral on the r.h.s of (4.13), by the change of variable $\hat{x}=\left(x-x_{C}\right) / R$ can be estimated as

$$
\begin{aligned}
& \iint_{A_{k}\left(x_{C}\right)} \frac{\left|\varphi_{R, x_{c}}(x)-\varphi_{R, x_{c}}(y)\right|^{\frac{N}{s}}}{|x-y|^{\left(s+\sigma_{k}\right) \frac{N}{s}}} \mathrm{~d} x \mathrm{~d} y \\
& =R^{2 N-\left(s+\sigma_{k}\right) \frac{N}{s}} \iint_{A_{k}(0)} \frac{\left|\varphi_{1,0}(\hat{x})-\varphi_{1,0}(\hat{y})\right|^{\frac{N}{s}}}{|\hat{x}-\hat{y}|^{\left(s+\sigma_{k}\right) \frac{N}{s}}} d \hat{x} d \hat{y} \\
& =R^{\left(s-\sigma_{k}\right) \frac{N}{s}} \iint_{A_{k}(0)} \frac{\left|\varphi_{1,0}(\hat{x})-\varphi_{1,0}(\hat{y})\right|^{\frac{N}{s}}}{|\hat{x}-\hat{y}|^{N+\sigma_{k} \frac{N}{s}}} d \hat{x} d \hat{y} \leq C R^{\left(s-\sigma_{k}\right) \frac{N}{s}} .
\end{aligned}
$$

For the second integral on the r.h.s of (4.13) we proceed decomposing it on the three sets (4.11).

Let $k=0$. When $(x, y) \in A_{0}\left(x_{C}\right)$ we have that $|x-y| \geq\left|y-x_{C}\right|-\left|x-x_{C}\right| \geq\left|y-x_{C}\right| / 2$ and therefore

$$
\begin{aligned}
& \iint_{A_{0}\left(x_{C}\right)} \frac{|w|_{\lambda}^{2_{s}^{*}}(y)}{|x-y|^{\frac{N-2 \sigma_{0}}{N-2 s}}} \mathrm{~d} x \mathrm{~d} y \leq C \iint_{A_{0}\left(x_{C}\right)} \frac{|w|_{\lambda}^{2_{s}^{*}}(y)}{\left|y-x_{C}\right|^{\frac{N-2 \sigma_{0}}{N-2 s}}} \mathrm{~d} x \mathrm{~d} y \\
& \leq C \int_{\mathbb{R}^{N}}\left(\int_{0}^{\left|y-x_{C}\right| / 2} \rho^{N-1} d \rho\right) \frac{|w|_{\lambda}^{2_{s}^{*}}(y)}{\left|y-x_{C}\right|^{\frac{N-2 \sigma_{0}}{N-2 s}}} \mathrm{~d} y \leq C \int_{\mathbb{R}^{N}}|w|_{\lambda}^{2_{s}^{*}}(y) \mathrm{d} y,
\end{aligned}
$$

with $C=C(N)$ a positive constant and where we used the fact that $\sigma_{0}=s$.

Let $k=1$. Recalling that $\sigma_{1} \in(0, s)$, we obtain

$$
\begin{aligned}
& \iint_{A_{1}\left(x_{C}\right)} \frac{|w|_{\lambda}^{2_{s}^{*}}(y)}{|x-y|^{\frac{N-2 \sigma_{1}}{N-2 s}}} \mathrm{~d} x \mathrm{~d} y \\
& \leq \int_{\mathbb{R}^{N}}\left(\int_{\mathbb{R}^{N} \backslash B_{R}(y)} \frac{1}{|x-y|^{\frac{N-2 \sigma_{1}}{N-2 s}}} \mathrm{~d} x\right)|w|_{\lambda}^{2_{s}^{*}}(y) \mathrm{d} y \\
& =\int_{\mathbb{R}^{N}}|w|_{\lambda}^{2_{s}^{*}}(y) \mathrm{d} y \cdot \int_{\mathbb{R}^{N} \backslash B_{R}(0)} \frac{1}{|\hat{x}|^{N+\frac{2 N\left(s-\sigma_{1}\right)}{N-2 s}}} \mathrm{~d} x \\
& =C R^{\frac{2 N\left(\sigma_{1}-s\right)}{N-2 s}} \int_{\mathbb{R}^{N}}|w|_{\lambda}^{2_{s}^{*}}(y) \mathrm{d} y,
\end{aligned}
$$

where in the last line we used the change of variable $\hat{x}=x-y$ and where $C=C\left(s, \sigma_{1}, N\right)$ is a positive constant.

Let $k=2$. Recalling that $\sigma_{2} \in(s, 1)$, we deduce

$$
\begin{aligned}
& \iint_{A_{2}\left(x_{C}\right)} \frac{|w|_{\lambda}^{2_{s}^{*}}(y)}{|x-y|^{\frac{N-2 \sigma_{2}}{N-2 s}}} \mathrm{~d} x \mathrm{~d} y \\
& \quad \leq \int_{\mathbb{R}^{N}}\left(\int_{B_{R}(y)} \frac{1}{|x-y|^{\frac{N-2 \sigma_{2}}{N-2 s}}} \mathrm{~d} x\right)|w|_{\lambda}^{2_{s}^{*}}(y) \mathrm{d} y
\end{aligned}
$$




$$
\begin{aligned}
& =\int_{\mathbb{R}^{N}}|w|_{\lambda}^{2_{s}^{*}}(y) \mathrm{d} y \cdot \int_{B_{R}(0)} \frac{1}{|\hat{x}|^{N-\frac{2 N\left(\sigma_{2}-s\right)}{N-2 s}}} \mathrm{~d} x \\
& =C R^{\frac{2 N\left(\sigma_{2}-s\right)}{N-2 s}} \int_{\mathbb{R}^{N}}|w|_{\lambda}^{2_{s}^{*}}(y) \mathrm{d} y,
\end{aligned}
$$

where $C=C\left(s, \sigma_{2}, N\right)$ is a positive constant.

Collecting (4.15), (4.16) and (4.17) we have that

$$
\iint_{A_{k}\left(x_{C}\right)} \frac{|w|_{\lambda}^{2_{s}^{*}}(y)}{|x-y|^{\frac{N-2 \sigma_{2}}{N-2 s}}} \mathrm{~d} x \mathrm{~d} y \leq C R^{\frac{2 N\left(\sigma_{k}-s\right)}{N-2 s}} \int_{\mathbb{R}^{N}}|w|_{\lambda}^{2_{s}^{*}}(y) \mathrm{d} y .
$$

From (4.13), using (4.14) and (4.18) it follows

$$
I_{11 k} \leq C R^{2\left(s-\sigma_{k}\right)}\left(R^{\frac{2 N\left(\sigma_{k}-s\right)}{N-2 s}} \int_{\mathbb{R}^{N}}|w|_{\lambda}^{2_{s}^{*}}(y) \mathrm{d} y\right)^{\frac{N-2 s}{N}} \leq C\left(\int_{\mathbb{R}^{N}}|w|_{\lambda}^{2_{s}^{*}}(y) \mathrm{d} y\right)^{\frac{N-2 s}{N}},
$$

where $C$ is a positive constant not depending on $R$. Finally from (4.12), we obtain

$$
\begin{aligned}
I_{11} & \leq C\left(\int_{\mathbb{R}^{N}}|w|_{\lambda}^{2_{s}^{*}}(y) \mathrm{d} y\right)^{\frac{N-2 s}{N}} \\
& =C\left(\int_{\mathbb{R}^{N} \backslash B_{R_{0}}(0)}|w|_{\lambda}^{2_{s}^{*}}(y) \mathrm{d} y+\int_{B_{R_{0}}(0)}|w|_{\lambda}^{2_{s}^{*}}(y) \mathrm{d} y\right)^{\frac{N-2 s}{N}} \leq C_{11},
\end{aligned}
$$

with $R_{0}$ given in the statement of Theorem 1.2 and where $C_{11}$ is a positive constant that does not depend on $R$ (and on $\varepsilon$ ). We point out that, in the last line of (4.20) we used the fact that $w_{\lambda}(x) \leq u(x), u \in L^{2_{s}^{*}}\left(\mathbb{R}^{N} \backslash B_{R_{0}}\right)$ and that, by (3.1), $w_{\lambda} \in L^{\infty}\left(B_{R_{0}}\right)$. To estimate the term $I_{12}$ in (4.10), we fix a radius $\hat{R}>R_{0}$ such that $\overline{B_{R_{0}} \cup R_{\lambda}\left(B_{R_{0}}\right)} \subset B_{\hat{R}}$. Therefore, using that (see (3.6)) $\varphi_{\varepsilon}^{\lambda}(x)=1$ in $\mathbb{R}^{N} \backslash B_{\hat{R}}$

$$
\begin{aligned}
I_{12} \leq & C \int_{\mathbb{R}^{N}} \int_{\mathbb{R}^{N}} \frac{\left|\varphi_{\varepsilon}(x)-\varphi_{\varepsilon}(y)\right|^{2} w_{\lambda}^{2}(y)}{|x-y|^{N+2 s}} \mathrm{~d} x \mathrm{~d} y \\
= & C \int_{B_{\hat{R}}} \int_{B_{\hat{R}}} \frac{\left|\varphi_{\varepsilon}(x)-\varphi_{\varepsilon}(y)\right|^{2} w_{\lambda}^{2}(y)}{|x-y|^{N+2 s}} \mathrm{~d} x \mathrm{~d} y \\
& +\int_{\mathbb{R}^{N} \backslash B_{\hat{R}}} \int_{B_{\hat{R}}} \frac{\left|\varphi_{\varepsilon}(x)-\varphi_{\varepsilon}(y)\right|^{2} w_{\lambda}^{2}(y)}{|x-y|^{N+2 s}} \mathrm{~d} x \mathrm{~d} y \\
& +\int_{B_{\hat{R}}} \int_{\mathbb{R}^{N} \backslash B_{\hat{R}}} \frac{\left|\varphi_{\varepsilon}(x)-\varphi_{\varepsilon}(y)\right|^{2} w_{\lambda}^{2}(y)}{|x-y|^{N+2 s}} \mathrm{~d} x \mathrm{~d} y \\
:= & \sum_{k=0}^{2} I_{12 k} .
\end{aligned}
$$

By Definition (3.1) we have that $w_{\lambda} \in L^{\infty}\left(B_{\hat{R}}\right)$. Thus, using (4.2) we obtain

$$
I_{120} \leq C \int_{B_{\hat{R}}} \int_{B_{\hat{R}}} \frac{\left|\varphi_{\varepsilon}(x)-\varphi_{\varepsilon}(y)\right|^{2}}{|x-y|^{N+2 s}} \mathrm{~d} x \mathrm{~d} y
$$




$$
\leq C \int_{\mathbb{R}^{N}} \int_{\mathbb{R}^{N}} \frac{\left|\phi_{\varepsilon}(x)-\phi_{\varepsilon}(y)\right|^{2}}{|x-y|^{N+2 s}} \mathrm{~d} x \mathrm{~d} y \leq C \varepsilon
$$

where, $A_{\hat{R}}:=R_{\lambda}\left(B_{\hat{R}}\right)$ and $C=C\left(\|u\|_{L^{\infty}\left(A_{\hat{R}}\right)}\right)$ is a positive constant. Similarly we also get

$$
I_{121} \leq C \varepsilon
$$

For the last term of (4.21) we argue splitting it in two terms:

$$
\begin{aligned}
I_{122}= & \int_{B_{\hat{R}}} \int_{B_{2 \hat{R}} \backslash B_{\hat{R}}} \frac{\left|\varphi_{\varepsilon}(x)-\varphi_{\varepsilon}(y)\right|^{2} w_{\lambda}^{2}(y)}{|x-y|^{N+2 s}} \mathrm{~d} x \mathrm{~d} y \\
& +\int_{B_{\hat{R}}} \int_{\mathbb{R}^{N} \backslash B_{2 \hat{R}}} \frac{\left|\varphi_{\varepsilon}(x)-\varphi_{\varepsilon}(y)\right|^{2} w_{\lambda}^{2}(y)}{|x-y|^{N+2 s}} \mathrm{~d} x \mathrm{~d} y .
\end{aligned}
$$

For the first term, as we did in (4.22), we have

$$
\int_{B_{\hat{R}}} \int_{B_{2 \hat{R}} \backslash B_{\hat{R}}} \frac{\left|\varphi_{\varepsilon}(x)-\varphi_{\varepsilon}(y)\right|^{2} w_{\lambda}^{2}(y)}{|x-y|^{N+2 s}} \mathrm{~d} x \mathrm{~d} y \leq C \varepsilon,
$$

with $C=C\left(\|u\|_{L^{\infty}\left(A_{2 \hat{R}}\right)}\right)$. For the second term we use Hölder inequality deducing

$$
\begin{aligned}
& \int_{B_{\hat{R}}} \int_{\mathbb{R}^{N} \backslash B_{2 \hat{R}}} \frac{\left|\varphi_{\varepsilon}(x)-\varphi_{\varepsilon}(y)\right|^{2} w_{\lambda}^{2}(y)}{|x-y|^{N+2 s}} \mathrm{~d} x \mathrm{~d} y \\
& \leq \int_{B_{\hat{R}}}\left(\left(\int_{\mathbb{R}^{N} \backslash B_{2 \hat{R}}} w_{\lambda}^{2_{s}^{*}}(y) \mathrm{d} y\right)^{\frac{N-2 s}{N}}\left(\int_{\mathbb{R}^{N} \backslash B_{2 \hat{R}}} \frac{\left|\varphi_{\varepsilon}(x)-\varphi_{\varepsilon}(y)\right|^{\frac{N}{s}}}{|x-y|^{\frac{(N+2 s) N}{2 s}}} \mathrm{~d} y\right)^{\frac{2 s}{N}}\right) \mathrm{d} x \\
& \quad \leq C\left(\int_{B_{\hat{R}}} \int_{\mathbb{R}^{N} \backslash B_{2 \hat{R}}} \frac{\left|\varphi_{\varepsilon}(x)-\varphi_{\varepsilon}(y)\right|^{\frac{N}{s}}}{|x-y|^{\frac{(N+2 s) N}{2 s}}} \mathrm{~d} y \mathrm{~d} x\right)^{\frac{2 s}{N}},
\end{aligned}
$$

with $C=C\left(s, N, \hat{R},\|u\|_{L^{2 *}\left(\mathbb{R}^{N} \backslash B_{2 \hat{R}}\right)},\|u\|_{L^{\infty}\left(A_{2 \hat{R}}\right)}\right)$. Since for all $(x, y) \in B_{\hat{R}} \times \mathbb{R}^{N} \backslash B_{2 \hat{R}}$, it follows that $|x-y| \geq \delta>0$, from (4.25) we infer that

$$
\int_{B_{\hat{R}}} \int_{\mathbb{R}^{N} \backslash B_{2 \hat{R}}} \frac{\left|\varphi_{\varepsilon}(x)-\varphi_{\varepsilon}(y)\right|^{2} w_{\lambda}^{2}(y)}{|x-y|^{N+2 s}} \mathrm{~d} x \mathrm{~d} y \leq C \int_{2 \hat{R}}^{\infty} \frac{1}{\rho^{\frac{N^{2}+2 s}{2 s}}} d \rho<+\infty
$$

and $C=C\left(s, N, \hat{R},\|u\|_{L^{2 *}\left(\mathbb{R}^{N} \backslash B_{2 \hat{R}}\right)},\|u\|_{L^{\infty}\left(A_{2 \hat{R}}\right)}\right)$. Using (4.22), (4.23) and (4.26), from (4.21) we deduce

$$
I_{12} \leq C_{12}(1+\varepsilon)
$$

Finally from (4.10), collecting (4.20) and (4.27) it follows

$$
I_{1} \leq C_{1}(1+\varepsilon)
$$

for some positive constant $C_{1}$. 
To estimate $I_{2}$ in (4.9) we use the mean value theorem and $\left(A_{f}^{2}\right)$. In fact

$$
\begin{aligned}
I_{2} & =\int_{\mathbb{R}^{N}}\left(\frac{f(u)-f\left(u_{\lambda}\right)}{u-u_{\lambda}}\right)\left(w_{\lambda}\right)^{2} \varphi_{\varepsilon}^{2} \varphi_{R, x_{c}}^{2} \mathrm{~d} x \\
& \leq 2 \int_{\Sigma_{\lambda}} f^{\prime}\left(\xi_{\lambda}\right)\left(w_{\lambda}\right)^{2} \varphi_{\varepsilon}^{2} \varphi_{R, x_{c}}^{2} \mathrm{~d} x \quad\left(\text { for } u<\xi_{\lambda}<u_{\lambda}\right) \\
& \leq 2 \int_{\Sigma_{\lambda}} f^{\prime}(u) w_{\lambda}^{2} \varphi_{\varepsilon}^{2} \varphi_{R, x_{c}}^{2} \mathrm{~d} x \quad \text { (since } f(\cdot) \text { is convex) } \\
& \leq 2 C_{f} \int_{\Sigma_{\lambda}} u^{2^{*}} \mathrm{~d} x \leq C_{2},
\end{aligned}
$$

where $C_{2}\left(f,\|u\|_{L^{2 *}\left(\mathbb{R}^{N} \backslash B_{R_{0}}\right)},\|u\|_{L^{\infty}\left(\Sigma_{\lambda} \cap B_{R_{0}}\right)}\right)$. Using (4.28) and (4.29) and redefining the constants, from (4.9) we have

$$
\int_{\mathbb{R}^{N}} \int_{\mathbb{R}^{N}} \frac{\left(w_{\lambda}(x)-w_{\lambda}(y)\right)^{2}}{|x-y|^{N+2 s}}\left(\frac{\varphi_{\varepsilon}^{2}(x) \varphi_{R, x_{c}}^{2}(x)+\varphi_{\varepsilon}^{2}(y) \varphi_{R, x_{c}}^{2}(y)}{2}\right) \mathrm{d} x \mathrm{~d} y \leq C(1+\varepsilon) .
$$

The thesis follows now by Fatou Lemma as (first) $\varepsilon$ tends to zero and (then) $R$ tends to infinity.

Proof of Theorem 1.2 We start the moving plane procedure by showing that for $\lambda<0$ and $|\lambda|$ large, we obtain that $u \leq u_{\lambda}$ in $\Sigma_{\lambda} \backslash R_{\lambda}(\Gamma)$. In fact using $\varphi:=w_{\lambda} \varphi_{\varepsilon}^{2} \varphi_{R, x_{c}}^{2}$ in Eq. (1.2) fulfilled by $u$ and in Eq. (4.3) fulfilled by $u_{\lambda}$, subtracting we get (see Eq. (4.4))

$$
\begin{aligned}
& \frac{1}{2} c_{N, s} \int_{\mathbb{R}^{N}} \int_{\mathbb{R}^{N}} \frac{\left(\left(u(x)-u_{\lambda}(x)\right)-\left(u(y)-u_{\lambda}(y)\right)\right)}{|x-y|^{N+2 s}} \\
& \quad \times\left(w_{\lambda}(x) \varphi_{\varepsilon}^{2}(x) \varphi_{R, x_{c}}^{2}(x)-w_{\lambda}(y) \varphi_{\varepsilon}^{2}(y) \varphi_{R, x_{c}}^{2}(y)\right) \mathrm{d} x \mathrm{~d} y \\
& \quad \leq \int_{\mathbb{R}^{N}}\left(\frac{f(u)-f\left(u_{\lambda}\right)}{u-u_{\lambda}}\right)\left(w_{\lambda}\right)^{2} \varphi_{\varepsilon}^{2} \varphi_{R, x_{c}}^{2} \mathrm{~d} x
\end{aligned}
$$

and then, as in (4.5), we have

$$
\begin{aligned}
& \frac{1}{2} c_{N, s} \int_{\mathbb{R}^{N}} \int_{\mathbb{R}^{N}} \frac{\left(w_{\lambda}(x)-w_{\lambda}(y)\right)\left(w_{\lambda}(x) \varphi_{\varepsilon}^{2}(x) \varphi_{R, x_{c}}^{2}(x)-w_{\lambda}(y) \varphi_{\varepsilon}^{2}(y) \varphi_{R, x_{c}}^{2}(y)\right)}{|x-y|^{N+2 s}} \mathrm{~d} x \mathrm{~d} y \\
& \quad \leq \int_{\mathbb{R}^{N}}\left(\frac{f(u)-f\left(u_{\lambda}\right)}{u-u_{\lambda}}\right)\left(w_{\lambda}\right)^{2} \varphi_{\varepsilon}^{2} \varphi_{R, x_{c}}^{2} \mathrm{~d} x .
\end{aligned}
$$

Using that $\varphi_{\varepsilon}^{2} \varphi_{R, x_{c}}^{2} \leq 1$ in all $\mathbb{R}^{N}$, it follows

$$
\begin{aligned}
& \int_{\mathbb{R}^{N}} \int_{\mathbb{R}^{N}} \frac{\left(w_{\lambda}(x) \varphi_{\varepsilon}^{2}(x) \varphi_{R, x_{c}}^{2}(x)-w_{\lambda}(y) \varphi_{\varepsilon}^{2}(y) \varphi_{R, x_{c}}^{2}(y)\right)^{2}}{|x-y|^{N+2 s}} \mathrm{~d} x \mathrm{~d} y \\
& \leq 2 \int_{\mathbb{R}^{N}} \int_{\mathbb{R}^{N}} \frac{\left(w_{\lambda}(x)-w_{\lambda}(y)\right)^{2}}{|x-y|^{N+2 s}} \mathrm{~d} x \mathrm{~d} y \\
& +4 \int_{\mathbb{R}^{N}} \int_{\mathbb{R}^{N}} \frac{\left(\varphi_{\varepsilon}(y) \varphi_{R, x_{c}}(y)-\varphi_{\varepsilon}(x) \varphi_{R, x_{c}}(x)\right)^{2} w_{\lambda}^{2}(y)}{|x-y|^{N+2 s}} \mathrm{~d} x \mathrm{~d} y
\end{aligned}
$$


and therefore, by Lemma 4.1, (4.9), (4.10) and (4.20)

$$
\int_{\mathbb{R}^{N}} \int_{\mathbb{R}^{N}} \frac{\left(w_{\lambda}(x) \varphi_{\varepsilon}^{2}(x) \varphi_{R, x_{c}}^{2}(x)-w_{\lambda}(y) \varphi_{\varepsilon}^{2}(y) \varphi_{R, x_{c}}^{2}(y)\right)^{2}}{|x-y|^{N+2 s}} \mathrm{~d} x \mathrm{~d} y \leq C,
$$

with $C$ is a positive constant not depending on $\varepsilon$ and $R$. Letting first $\varepsilon$ to zero and then $R$ to infinity, using Lemma 4.1 and (4.31), the 1.h.s of (4.30) by weak convergence goes to

$$
\frac{1}{2} c_{N, s} \int_{\mathbb{R}^{N}} \int_{\mathbb{R}^{N}} \frac{\left(w_{\lambda}(x)-w_{\lambda}(y)\right)^{2}}{|x-y|^{N+2 s}} \mathrm{~d} x \mathrm{~d} y
$$

By $\left(A_{f}^{2}\right)$ and Lemma 4.1, the r.h.s of (4.30), by the dominate convergence Theorem goes to

$$
\int_{\mathbb{R}^{N}}\left(\frac{f(u)-f\left(u_{\lambda}\right)}{u-u_{\lambda}}\right)\left(w_{\lambda}\right)^{2} \mathrm{~d} x .
$$

Hence, (4.30) becomes

$$
\frac{1}{2} c_{N, s} \int_{\mathbb{R}^{N}} \int_{\mathbb{R}^{N}} \frac{\left(w_{\lambda}(x)-w_{\lambda}(y)\right)^{2}}{|x-y|^{N+2 s}} \mathrm{~d} x \mathrm{~d} y \leq \int_{\mathbb{R}^{N}}\left(\frac{f(u)-f\left(u_{\lambda}\right)}{u-u_{\lambda}}\right) w_{\lambda}^{2} \mathrm{~d} x .
$$

Using $\left(A_{f}^{2}\right)$ and Hölder inequality, it follows

$$
\begin{aligned}
& \frac{1}{2} c_{N, s} \int_{\mathbb{R}^{N}} \int_{\mathbb{R}^{N}} \frac{\left(w_{\lambda}(x)-w_{\lambda}(y)\right)^{2}}{|x-y|^{N+2 s}} \mathrm{~d} x \mathrm{~d} y \\
& \leq \int_{\mathbb{R}^{N}}\left(\frac{f(u)-f\left(u_{\lambda}\right)}{u-u_{\lambda}}\right) w_{\lambda}^{2} \mathrm{~d} x \\
& \leq 2 C_{f} \int_{\Sigma_{\lambda}} u^{2_{s}^{*}-2} w_{\lambda}^{2} \mathrm{~d} x \\
& \leq 2 C_{f}\left(\int_{\Sigma_{\lambda}} u^{2_{s}^{*}} \mathrm{~d} x\right)^{\frac{2_{s}^{*}-2}{2_{s}^{*}}}\left(\int_{\Sigma_{\lambda}} w_{\lambda}^{2_{s}^{*}} \mathrm{~d} x\right)^{\frac{2}{2_{s}^{*}}} \\
& \leq \frac{4 C_{f}}{S_{N, s} c_{N, s}}\left(\int_{\Sigma_{\lambda}} u^{2_{s}^{*}} \mathrm{~d} x\right)^{\frac{2_{s}^{*}-2}{2_{s}^{*}}} \int_{\mathbb{R}^{N}} \int_{\mathbb{R}^{N}} \frac{\left(w_{\lambda}(x)-w_{\lambda}(y)\right)^{2}}{|x-y|^{N+2 s}} \mathrm{~d} x \mathrm{~d} y,
\end{aligned}
$$

where the last inequality follows from Theorem 2.1 . Recalling that $u \in L^{2_{s}^{*}}\left(\mathbb{R}^{N} \backslash B_{R_{0}}\right.$ ), with $\Gamma \subset\left\{x_{1}=0\right\} \cap B_{R_{0}}$ we deduce that we can take $\lambda<0$, with $|\lambda|$ large, in such a way that

$$
\frac{4 C_{f}}{S_{N, s} c_{N, s}}\left(\int_{\Sigma_{\lambda}} u^{2_{s}^{*}} \mathrm{~d} x\right)^{\frac{2_{s}^{*}-2}{2 *}}<\frac{1}{4} c_{N, s} .
$$

A contradiction occurs by (4.32) unless

$$
\int_{\mathbb{R}^{N}} \int_{\mathbb{R}^{N}} \frac{\left(w_{\lambda}(x)-w_{\lambda}(y)\right)^{2}}{|x-y|^{N+2 s}} \mathrm{~d} x \mathrm{~d} y=0,
$$

that is $u \leq u_{\lambda}$ in $\Sigma_{\lambda}$. Let us now set

$$
\Lambda_{0}=\left\{\lambda<0: u \leq u_{t} \text { in } \Sigma_{t} \backslash R_{t}(\Gamma) \text { for all } t \in(-\infty, \lambda]\right\}
$$

and

$$
\lambda_{0}=\sup \Lambda_{0}
$$


that is well defined since we showed that $\Lambda_{0}$ is not empty. To prove our result we have to show that $\lambda_{0}=0$. To prove this we assume that $\lambda_{0}<0$ and we reach a contradiction by proving that $u \leq u_{\lambda_{0}+\tau}$ in $\Sigma_{\lambda_{0}+\tau} \backslash R_{\lambda_{0}+\tau}(\Gamma)$ for any $0<\tau<\bar{\tau}$ for some small $\bar{\tau}>0$. By continuity of $u$ in $\mathbb{R}^{N} \backslash \Gamma$, we know that $u \leq u_{\lambda_{0}}$ in $\Sigma_{\lambda_{0}} \backslash R_{\lambda_{0}}(\Gamma)$. By the strong maximum principle ([17, Proposition 3.6]) we deduce that $u<u_{\lambda_{0}}$ in $\Sigma_{\lambda_{0}} \backslash R_{\lambda_{0}}(\Gamma)$. Here we use that a symmetry position before the limiting position (namely $u=u_{\lambda_{0}}$ in $\Sigma_{\lambda_{0}} \backslash R_{\lambda_{0}}(\Gamma)$ ) is not possible, if $\lambda_{0}<0$, since in this case $u$ should be singular on $R_{\lambda_{0}}(\Gamma)$. For $\delta>0$ that will be chosen small later on, we consider a compact set $K_{\delta} \subset \Sigma_{\lambda_{0}} \backslash R_{\lambda_{0}}(\Gamma)$ such that

$$
\int_{\Sigma_{\lambda_{0}+\bar{\tau} \backslash K_{\delta}}} u^{2_{s}^{*}} \leq \delta .
$$

By uniform continuity, we can take $\bar{\tau}$ small such that $u<u_{\lambda_{0}+\tau}$ in $K_{\delta}$ for any $0<\tau<\bar{\tau}$. Now we repeat verbatim the arguments used at the beginning of this proof, using the test function $\varphi:=w_{\lambda_{0}+\tau} \varphi_{\varepsilon}^{2} \varphi_{R, x_{c}}^{2}$ in Eq. (3.7) fulfilled by $u$ and in Eq. (4.3) fulfilled by $u_{\lambda}$. Taking the limits, as in (4.32), we have

$$
\begin{aligned}
& \frac{1}{2} c_{N, s} \int_{\mathbb{R}^{N}} \int_{\mathbb{R}^{N}} \frac{\left(w_{\lambda}(x)-w_{\lambda}(y)\right)^{2}}{|x-y|^{N+2 s}} \mathrm{~d} x \mathrm{~d} y \\
& \leq \int_{\mathbb{R}^{N}}\left(\frac{f(u)-f\left(u_{\lambda}\right)}{u-u_{\lambda}}\right) w_{\lambda}^{2} \mathrm{~d} x \\
& \leq 2 C_{f} \int_{\Sigma_{\lambda_{0}+\tau} \backslash K_{\delta}} u^{2_{s}^{*}-2} w_{\lambda}^{2} \mathrm{~d} x \\
& \leq 2 C_{f}\left(\int_{\Sigma_{\lambda_{0}+\tau} \backslash K_{\delta}} u^{2_{s}^{*}} \mathrm{~d} x\right)^{\frac{2_{s}^{*}-2}{2_{s}^{*}}}\left(\int_{\Sigma_{\lambda_{0}+\tau} \backslash K_{\delta}} w_{\lambda}^{2_{s}^{*}} \mathrm{~d} x\right)^{\frac{2}{2_{s}^{*}}} \\
& \leq \frac{4 C_{f}}{S_{N, s} c_{N, s}}\left(\int_{\Sigma_{\lambda_{0}+\tau} \backslash K_{\delta}} u^{2_{s}^{*}} \mathrm{~d} x\right)^{\frac{2_{s}^{*}-2}{2_{s}^{*}}} \int_{\mathbb{R}^{N}} \int_{\mathbb{R}^{N}} \frac{\left(w_{\lambda}(x)-w_{\lambda}(y)\right)^{2}}{|x-y|^{N+2 s}} \mathrm{~d} x \mathrm{~d} y .
\end{aligned}
$$

Now we chose $\delta$ small in such a way that

$$
\begin{aligned}
\frac{4 C_{f}}{S_{N, s} c_{N, s}}\left(\int_{\Sigma_{\lambda_{0}+\tau} \backslash K_{\delta}} u^{2_{s}^{*}} \mathrm{~d} x\right)^{\frac{2_{s}^{*}-2}{2_{s}^{*}}} & \leq \frac{4 C_{f}}{S_{N, s} c_{N, s}}\left(\int_{\Sigma_{\lambda_{0}+\bar{\tau} \backslash K_{\delta}}} u^{2_{s}^{*}} \mathrm{~d} x\right)^{\frac{2_{s}^{*}-2}{2_{s}^{*}}} \\
& <\frac{1}{4} c_{N, s},
\end{aligned}
$$

obtaining the desired contradiction by (4.33) and showing that $\lambda_{0}=0$. The symmetry of the solution follows now performing the moving plane method in the opposite direction. The monotonicity of the solution is implicit in the technique.

If $u$ has only a nonremovable singularity at the origin, then the solution is radial and radially decreasing about the origin. This follows applying the moving plane procedure in any direction $v \in \mathbb{S}^{1}$ of $\mathbb{R}^{N}$.

\section{References}

1. Adams, R.A.: Sobolev Spaces. Pure and Applied mathematics, vol. 65. Academics Press, New York (1975) 
2. Alexandrov, A.D.: A characteristic property of the spheres. Ann. Mat. Pura Appl. 58, 303-354 (1962)

3. Barrios, B., Montoro, L., Sciunzi, B.: On the moving plane method for nonlocal problems in bounded domains. J. Anal. Math. arXiv:1405.5402 (to appear)

4. Berestycki, H., Nirenberg, L.: On the method of moving planes and the sliding method. Bolletin Soc. Brasil. de Mat Nova Ser. 22, 1-37 (1991)

5. Cabré, X., Sire, Y.: Nonlinear equations for fractional Laplacians II: existence, uniqueness, and qualitative properties of solutions. Trans. Amer. Math. Soc. 367(2), 911-941 (2015)

6. Caffarelli, L., Li, Y.Y., Nirenberg, L.: Some remarks on singular solutions of nonlinear elliptic equations. II: symmetry and monotonicity via moving planes. Advances in geometric analysis, 97105, Adv. Lect. Math. (ALM), 21, Int. Press, Somerville, MA (2012)

7. Chen, W., Li, C., Ou, B.: Qualitative properties of solutions for an integral equation. Discrete Contin. Dyn. Syst. 12(2), 347-354 (2005)

8. Chen, W., Li, C., Ou, B.: Classification of solutions for an integral equation. Commun. Pure Appl. Math. 59, 330-343 (2006)

9. Di Nezza, E., Palatucci, G., Valdinoci, E.: Hitchhiker's guide to the fractional Sobolev spaces. Bull. Sci. Math. 136(5), 521-573 (2012)

10. Dipierro, S., Valdinoci, E.: A density property for fractional weighted Sobolev Spaces. Atti Accad. Naz. Lincei Rend. Lincei Mat. Appl. 26(4), 397-422 (2015)

11. Dipierro, S., Palatucci, G., Valdinoci, E.: Existence and symmetry results for a Schrödinger type problem involving the fractional Laplacian. Le Matematiche (Catania) 68(1), 201-216 (2013)

12. Dipierro, S., Montoro, L., Peral, I., Sciunzi, B.: Qualitative properties of positive solutions to nonlocal critical problems involving the Hardy-Leray potential. Calc. Var. Part. Differ. Equ. 55(4), 99 (2016)

13. Fall, M.M.: Semilinear elliptic equations for the fractional Laplacian with Hardy potential. arXiv:1109.5530v4 (preprint)

14. Felmer, P., Wang, Y.: Radial symmetry of positive solutions to equations involving the fractional Laplacian. Commun. Contemp. Math. 16(1), 1350023-1350024 (2014)

15. Gidas, B., Ni, W.M., Nirenberg, L.: Symmetry and related properties via the maximum principle. Commun. Math. Phys. 68, 209-243 (1979)

16. Jarohs, S., Weth, T.: Asymptotic symmetry for a class of nonlinear fractional reaction-diffusion equations. Discrete Contin. Dyn. Syst. 34(6), 2581-2615 (2014)

17. Jarohs, S., Weth, T.: Symmetry via antisymmetric maximum principles in nonlocal problems of variable order. Ann. Mat. Pura Appl. 195(1), 273-291 (2016)

18. Landkof, N.: Foundations of modern potential theory. Die Grundlehren der mathematischen Wissenschaften, vol. 180. Springer, New York (1972)

19. Lieb, E.: Sharp constants in the Hardy-Littlewood-Sobolev and related inequalities. Ann. Math. 118(2), 349-374 (1983)

20. Ma, L., Chen, D.: Radial symmetry and monotonicity for an integral equation. J. Math. Anal. Appl. 342, 943-949 (2008)

21. Merchán, S., Montoro, L., Peral, I., Sciunzi, B.: Existence and qualitative properties of solutions to a quasilinear elliptic equation involving the Hardy-Leray potential. Ann. Inst. H. Poincaré Anal. Non Linéaire 31(1), 1-22 (2014)

22. Serrin, J.: A symmetry problem in potential theory. Arch. Ration. Mech. Anal 43(4), 304-318 (1971)

23. Sciunzi, B.: On the moving plane method for singular solutions to semilinear elliptic equations. J. Math. Pures Appl. 108(1), 111-123 (2017)

24. Sire, Y., Valdinoci, E.: Fractional Laplacian phase transitions and boundary reactions: a geometric inequality and a symmetry result. J. Funct. Anal. 256(6), 1842-1864 (2009)

25. Silvestre, L.: Regularity of the obstacle problem for a fractional power of the Laplace operator. Commun. Pure Appl. Math. 60(1), 67-112 (2007)

26. Stein, E.M.: Harmonic Analysis: Real-Variable Methods, Orthogonality, and Oscillatory Integrals. Mathematical Series Monographs in Harmonic Analysis III, vol. 43. Princeton University Press, Princeton (1993)

27. Stein, E.M.: Singular Integrals and Differentiability Properties of Functions. Princeton Mathematical Series, vol. 30. Princeton University Press, Princeton (1970)

28. Terracini, S.: On positive entire solutions to a class of equations with a singular coefficient and critical exponent. Adv. Differ. Equ. 1, 241-264 (1996)

29. Valdinoci, E.: From the long jump random walk to the fractional Laplacian. Bol. Soc. Esp. Mat. Apl. SeMA 49, 33-44 (2009)

30. Warma, M.: The fractional relative capacity and the fractional Laplacian with Neumann and Robin boundary conditions on open sets. Potential Anal. 42, 499-547 (2015) 